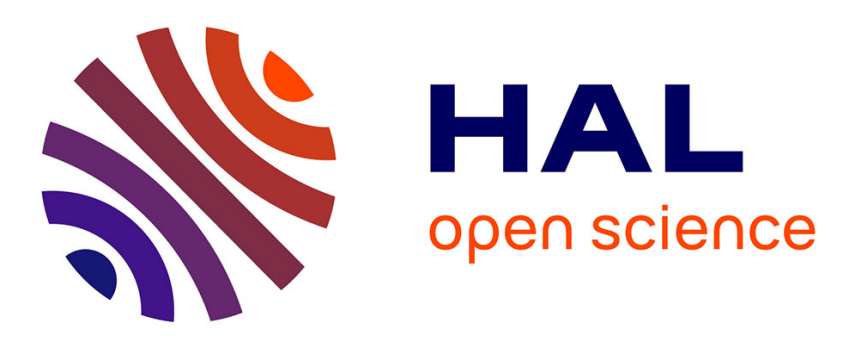

\title{
Thin functional zeolite layer supported on infrared resonant nano-antennas for fast detection of benzene traces
}

Javier Nunez, Arjen Boersma, Julien Grand, Svetlana Mintova, Beniamino

Sciacca

\section{To cite this version:}

Javier Nunez, Arjen Boersma, Julien Grand, Svetlana Mintova, Beniamino Sciacca. Thin functional zeolite layer supported on infrared resonant nano-antennas for fast detection of benzene traces. Advanced Functional Materials, 2021, 10.1002/adfm.202101623 . hal-03093749v2

\section{HAL Id: hal-03093749 \\ https://hal.science/hal-03093749v2}

Submitted on 14 Apr 2021

HAL is a multi-disciplinary open access archive for the deposit and dissemination of scientific research documents, whether they are published or not. The documents may come from teaching and research institutions in France or abroad, or from public or private research centers.
L'archive ouverte pluridisciplinaire HAL, est destinée au dépôt et à la diffusion de documents scientifiques de niveau recherche, publiés ou non, émanant des établissements d'enseignement et de recherche français ou étrangers, des laboratoires publics ou privés. 


\section{WILEY-VCH}

\section{Thin functional zeolite layer supported on infrared resonant nano- antennas for the detection of benzene traces}

Javier Nunez ${ }^{1}$, Arjen Boersma ${ }^{1}$, Julien Grand ${ }^{2}$, Svetlana Mintova ${ }^{2}$ and Beniamino Sciacca ${ }^{3 *}$

${ }^{1}$ MSC J. Nunez, Dr. A. Boersma

TNO Materials Solution, High Tech Campus 25, Eindhoven, Netherlands

2Dr. J. Grand, Dr. S. Mintova

Normandie Université, ENSICAEN, UNICAEN, CNRS, LCS, 14000 Caen, France

${ }^{3}$ Dr. Beniamino Sciacca

Aix-Marseille University, CNRS, CINaM, 13288 Marseille, France

E-mail: beniamino.sciacca@univ-amu.fr

Keywords: Resonant nano-antennas, Zeolite coating, Hybrid SEIRA material, VOCs, Benzene traces, Sensing

Infrared absorption spectroscopy is a powerful analytical tool that enables the identification of molecular species. The sensitivity of this technique, that is strongly limited by the small absorption cross-section of molecular vibration, can be greatly improved by resonant interaction with nano-antennas, via the surface enhanced infrared absorption (SEIRA) mechanism. Yet, most of the examples of SEIRA concerns solid-state molecular layers adsorbed on the nano-antennas, while the detection of gas traces still remains elusive, due to the spatial extent of the near-field that is used to resonantly amplify the molecular vibrations. Here we demonstrate a hybrid system composed of a plasmonic nano-antenna array coupled with nanosized zeolite coating for detection of volatile organic compounds in the near field of the resonators. The concerted action of the coating and the nano-antennas enabled us to detect record traces of benzene ( $25 \mathrm{ppb}$ ) within 10 minutes. This approach may ultimately allow the fabrication of a compact system for rapid detection of pharmaceutical and bio- compounds with high sensitivity and high selectivity. 


\section{WILEY-VCH}

\section{Introduction}

The detection of volatile organic compounds (VOC) at trace levels ( $\mathrm{ppb}$ to $\mathrm{ppm}$ ) is of extreme importance in the field of environmental monitoring for health related matters. The European Air Quality Directive defines as mandatory the monitoring of certain VOCs, such as benzene in ambient conditions and guideline values are provided by the World Health Organization with an associated risk. The classical method for such measurements is gas chromatography, which is time-consuming, expensive and not portable. Portable low-cost sensors have been developed for specific targets and are commercially available. Typically, these sensors either monitor changes such as conductance, strain or absorption in a responsive material such as a polymer, or monitor the potential or the current in a chemical reaction [1]. These approaches can be very sensitive for a specific VOC targeting a concentration in the range from ppb to sub ppb, but they either lack versatility (developed for one specific target) and/or selectivity (discern a target in complex and unknown mixtures).

For a sensor to be able to detect a specific VOC in the ppb range, the sensing layer, and often the entire device, has to be designed and tailored towards a target substance (an analyte). Thereby, this approach hampers the versatility of the sensor, in the sense that the same sensor cannot be used to detect other analytes, since, in principle, the transducing mechanism is responsive only (or to a larger extent) to the targeted analyte. This is a consequence of detecting a target substance indirectly, i.e. via an intermediate responsive material that imparts selectivity to the sensor, which is essential in real life applications.

Spectroscopic techniques in the mid infrared (3-20 um) are able to detect directly the unique fingerprints of molecular transitions, therefore offering a valid alternative to the aforementioned approaches, in terms of versatility and selectivity. The target substance with 


\section{WILEY-VCH}

an active IR vibration, can be detected from the unique fingerprint of its vibrational transitions, without the need for an intermediate transducer, or any prior knowledge.

However, versatility (ability to detect any species) and sensitivity (ability to detect low concentrations) are often incompatible in systems with a small footprint (cheap and portable). In fact, while IR spectroscopy allows for identification of chemical species in gas, liquid and solid media, the light-matter interaction is extremely weak, thereby requiring long optical paths to boost the signal to noise ratio for applications where detection at ppb levels is required. This is due to the fact that the absorption cross-section of molecules in the IR is extremely small, being proportional to $\sigma_{\text {abs }} \propto a^{3} / \lambda$, with $a$ being the molecule characteristic size [2]. In the specific case of benzene the absorption cross-section of IR vibrational modes for one molecule is in the range of $\sigma_{a b s} \approx 1-10 \times 10^{-20} \mathrm{~cm}^{2}$ [3]. Therefore, a large amount of molecules is needed to yield a signal above the noise level, in accordance with the LambertBeer's relationship. To keep a small footprint, it has been proposed to coat mid-IR waveguides or optical fibers with materials able to adsorb species of interest, thereby locally increasing the concentration $[4,5]$. However, this approach often lacks in sensitivity that is required in many applications. One example is the monitoring of benzene at ppb or sub ppb levels, that remains challenging even if a separate pre-concentration step is employed [6].

The use of resonant nano-antennas has proven to be effective in enhancing light absorption by organic molecules via the near field enhancement effect (Surface Enhanced Infrared Absorption - SEIRA) [7, 8]. The increase of sensitivity by 3 to 5 orders of magnitude compared to traditional IR absorption spectroscopy on films was reported [9]. Antennas are designed to have the resonance overlapping with the vibrational transition of target molecules as shown in Figure 1a [10,11]. This approach is very successful for detecting the vibrational fingerprint of solid state monolayers covering the nano-antennas. In such systems, the target 


\section{WILEY-VCH}
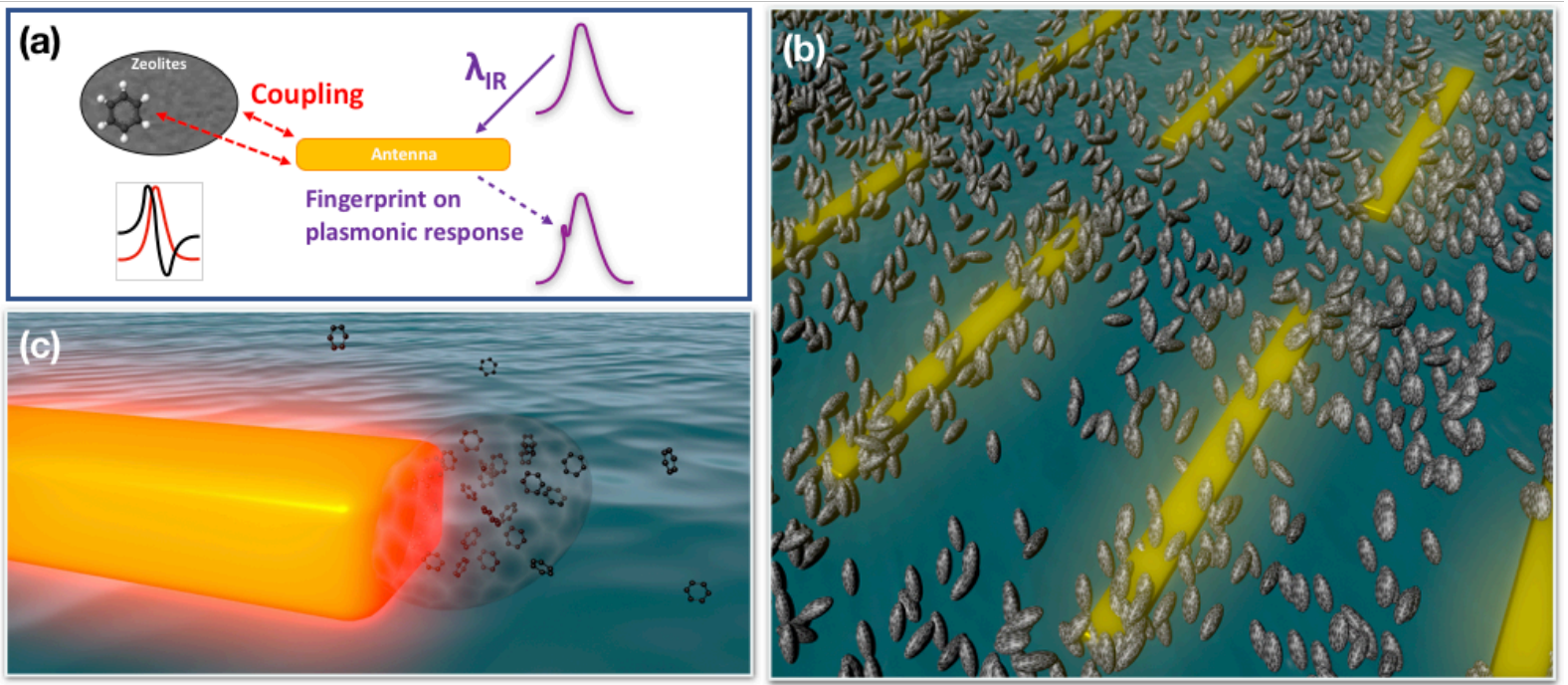

Figure 1: (a) Surface-Enhanced Infrared Absorption (SEIRA) concept: near-field coupling between molecular/phonon transitions and optical resonances enables the amplification of the fingerprint. (b) Gold nanorods array coated by zeolite nanocrystals able to trap benzene in regions where the near field enhancement is the highest (c).

molecules have to be very close to the antenna surface, where the field enhancement is the highest [12]. However, for low concentrated gases the probability of a molecule transiting within the strong field enhancement region can be very low. As an example, at a concentration of $25 \mathrm{ppb}$, there is roughly 1 molecule of benzene in a volume of $1 \mu \mathrm{m}^{3}$, whereas the mode volume of standard SEIRA nano-antennas is many orders of magnitude smaller $\left(\sim 10^{-3} \mu \mathrm{m}^{3}\right)$. Therefore, for low concentrated volatile compounds that do not show significant adsorption on the antennas, the SEIRA approach alone is not suitable.

In this communication, we present a new approach that enables to extend the SEIRA towards the detection of gases at very low concentrations. The quick detection of benzene in the range of 20-30 ppb using a thin functional layer, and the capability to discriminate benzene from other VOCs such as toluene is demonstrated. To the best of our knowledge this sets a record for benzene detection using IR spectroscopy without external pre-concentration steps, considering that the concentrating role of the zeolite occurs in real-time with the measurement itself, as a one-step process. In addition, these values represent a great enhancement with respect to the low ppm range detection limit reported in the literature by using IR 


\section{WILEY-VCH}

spectroscopy without a separate pre-concentration step, or with respect to the hundreds of ppb reported with IR tunable lasers $[6,13,14]$.

\section{Results and discussion}

\subsection{Zeolite concentrating coating}

The development of an IR device for fast VOC detection of analytes in the ppb range requires a concerted approach in which the target molecules are trapped in close proximity of resonant nano-antennas hot spots, where the SEIRA can effectively take place. This can be achieved by coating the nano-antenna with a porous material able to capture and retain the analytes of interest. The porous material could be either organics such as polymers, hybrid such as metalorganic frameworks (MOF) or inorganic, such as zeolites or clays. Similar approaches were recently proposed by combining SEIRA nano-antennas with a MOF coating able to adsorb selectively $\mathrm{CO}_{2}$ or $\mathrm{CH}_{4}$. An improved detection limit for $\mathrm{CO}_{2}$ in the order of several tens of ppm was reported $[15,16,17]$. This approach is limited to a certain extent due to the fact that the hybrid material used possesses many vibrational modes overlapping with molecules of interest (analytes).

Thus the desired 'concentrating' coating should be suited to adsorb molecules with very low concentration and be compatible with the integration on the resonant nano-antennas. Zeolites have little overlap of vibrational bands of the targeted molecules thanks to the pure inorganic nature [18]. Additionally, the zeolite with nanosized crystals enabled their deposition in a sub-100 nm zeolite film at low temperature via spin coating, which is compatible with resonant nano-antennas $[19,20]$. Among the available zeolites, the selection made in our study was based on (i) the pore size of the zeolites (that should be large enough to host benzene), (ii) the hydrophobicity (to retain little water and adsorb predominantly VOCs), (iii) the Henry's constant, i.e. to adsorb VOCs at very low partial pressure [21], and (iv) the 


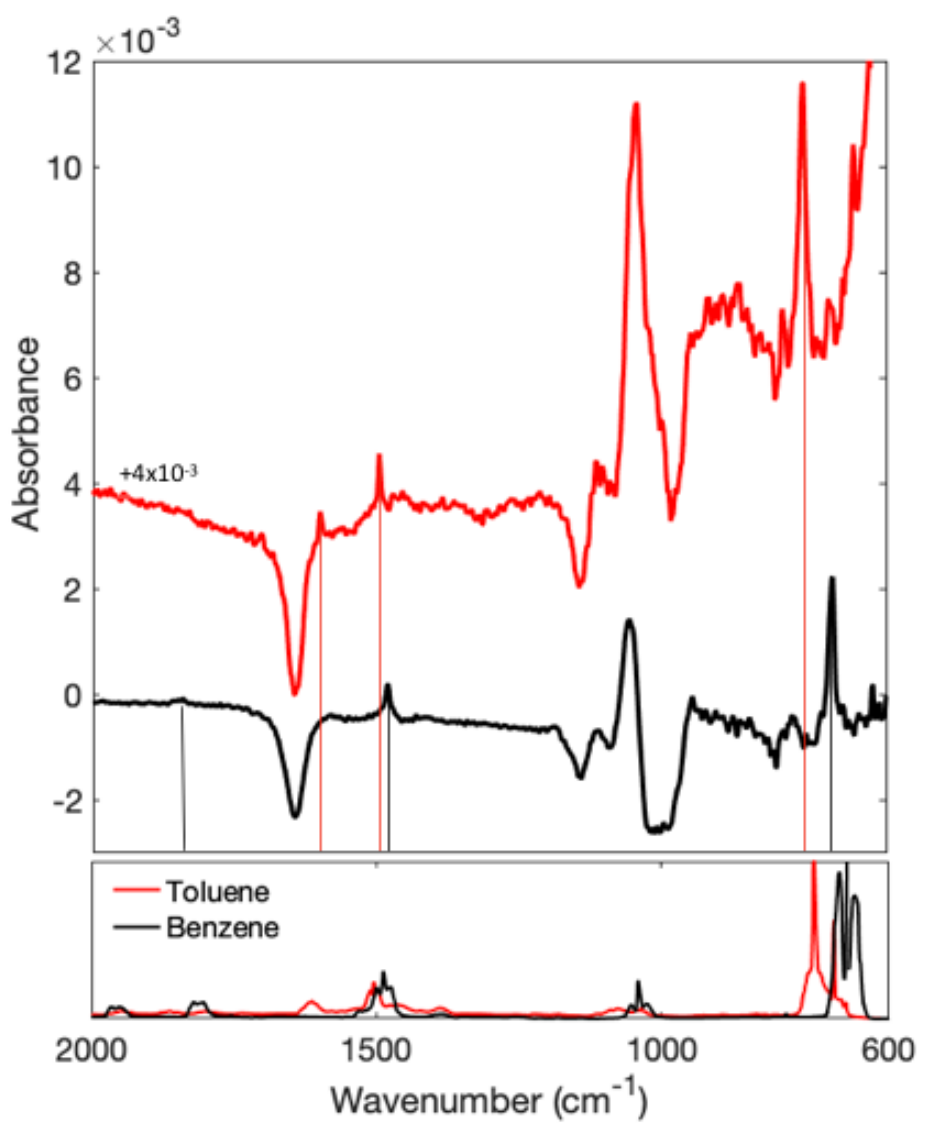

Figure 2: IR spectra of benzene (black solid line) and toluene (red solid line) adsorbed on FAU coating. Bottom panel: the IR spectra of toluene and benzene in gas phase.

availability of the zeolites with nanosized dimensions stable in coating suspensions. Faujasite zeolite (sample denoted as FAU), [22] with a suitable pore diameter $(0.72 \mathrm{~nm})$ to adsorb benzene $(0.5 \mathrm{~nm})$ [23] and a crystal size of $\sim 20 \mathrm{~nm}$, was selected for the current work.

Figure 2 shows the IR spectrum of benzene (black solid line) upon adsorption on FAU nanozeolite layer, while its spectrum in the gas form is shown in the bottom panel. Although the spectral shape of the molecular vibrations differs after adsorption, the peaks position and relative intensity are approximately unchanged. In particular, the characteristic aromatic $\mathrm{C}=\mathrm{C}$ stretching $\left(1480 \mathrm{~cm}^{-1}\right)$ and the out of plane C-H vibration $\left(694 \mathrm{~cm}^{-1}\right)$ are clearly visible. It's important to notice a negative peak at $1645 \mathrm{~cm}^{-1}$, corresponding to the loss of water still adsorbed in the zeolite structure despite extensive flushing with $\mathrm{N}_{2}$ at room temperature. There are strategies, such as thermal desorption in vacuum, that are routinely used to desorb residual water from the zeolite pores, but those were not compatible with our experimental 


\section{WILEY-VCH}

setup. Although the presence of residual water in the pores has possibly a negative impact on the performance, it reproduces more realistic conditions.

Benzene exposure promotes rapid water desorption, probably due to its higher affinity towards the coating surface. The negative peaks at $1140 \mathrm{~cm}^{-1}$ and $1000 \mathrm{~cm}^{-1}$ and the positive peak at $1053 \mathrm{~cm}^{-1}$ fall in the region of the T-O-T band of FAU (Figure $4 \mathbf{d}$, blue curve), that is modulated by the desorption of water and adsorption of benzene $(\mathrm{C}-\mathrm{H}$ in plane deformation at $\left.1040 \mathrm{~cm}^{-1}\right)$.

Next, toluene was selected as an analyte to evaluate the selectivity of the sensors, i.e., the capability to distinguish between molecules with very similar structures. We present in Figure 2 the IR spectra of toluene adsorbed in the FAU zeolite coating and in the gas phase. One can still discern between benzene and toluene even after adsorption in the zeolite coating. The use of zeolite coatings does not affect the quality of the IR spectra.

We evaluated the performance of the FAU coating by comparing the absorbance of the aromatic $\mathrm{C}=\mathrm{C}$ stretching $\left(1480 \mathrm{~cm}^{-1}\right)$ for a coating with a defined thickness with that of benzene in the gas phase for a given optical path. The concentration factor $\boldsymbol{\alpha}$ was defined as:

$$
\alpha=\frac{C_{\text {ref }} d_{\text {cell }}}{A_{\text {ref }}} \frac{A_{\text {sat }}}{C_{0} t_{\text {coat }}}
$$

$A_{\text {ref }}$ is the absorbance (for a specific peak) measured at concentration $C_{\text {ref }}$ if no coating is used, and $d_{\text {cell }}$ is the optical path of the cell. $A_{\text {sat }}$ is the absorbance (of the same peak) measured in equilibrium conditions for a concentration $\mathrm{C}_{0}$ when a zeolite coating with a thickness $t_{\text {coat }}$ is present. For the FAU zeolite coating, $\boldsymbol{\alpha}=6 \cdot 10^{8}$ was estimated from Equation 1, meaning that in the linear regime (coating not saturated) under steady-state conditions, the concentration of benzene inside the coating is more than eight orders of magnitude higher 


\section{WILEY-VCH}

than outside (see SI for further details). The interactions are reversible upon $\mathrm{N}_{2}$ delivery to the sensor, and the benzene desorbed from the zeolite coating (Figure S7).

\subsection{SEIRA resonant nano-antennas}

After identifying the best performing coating among those explored, we looked at a suitable nano-antennas design that is also a critical aspect of a device. Among the many possible designs, we fabricated gold nanorods in a phased array configuration. The individual nanorod is a nanoantenna itself with a resonance wavelength that depends on its characteristic size and on the refractive index of the supporting substrate. It is possible to use approximative scaling rules that predict the resonance wavelength from the length of the nanorods, given that nanorod width and height set [24]. Under some assumptions, a linear positive correlation of the resonance wavelength with the nanorod length is predicted. Therefore, nanorods are highly versatile, since they can be tuned to support resonances at any wavelength, by simply adjusting their length. By engineering the collective interaction in nanorod arrays, light scattered from individual nanorod can interfere constructively to yield a larger near field enhancement, and therefore a larger extinction in the far-field. The design of ordered arrays of nanorods has been shown to improve by one order of magnitude their performance compared to random arrays [25].

Resonant nano-antennas consisting of gold nanorods arrays where prepared by e-beam lithography on a ZnSe substrate that remains fairly transparent to IR radiation until $15 \mu \mathrm{m}$, where VOCs possess the characteristic vibrational modes. Numerical simulations were employed to design a set of phased arrays resonant with several characteristic vibrational modes of benzene. The dimensions of nanorods and the periodicity of the array were chosen to maximize the near field enhancement. Figure 3a shows a representative SEM image of the 


\section{WILEY-VCH}

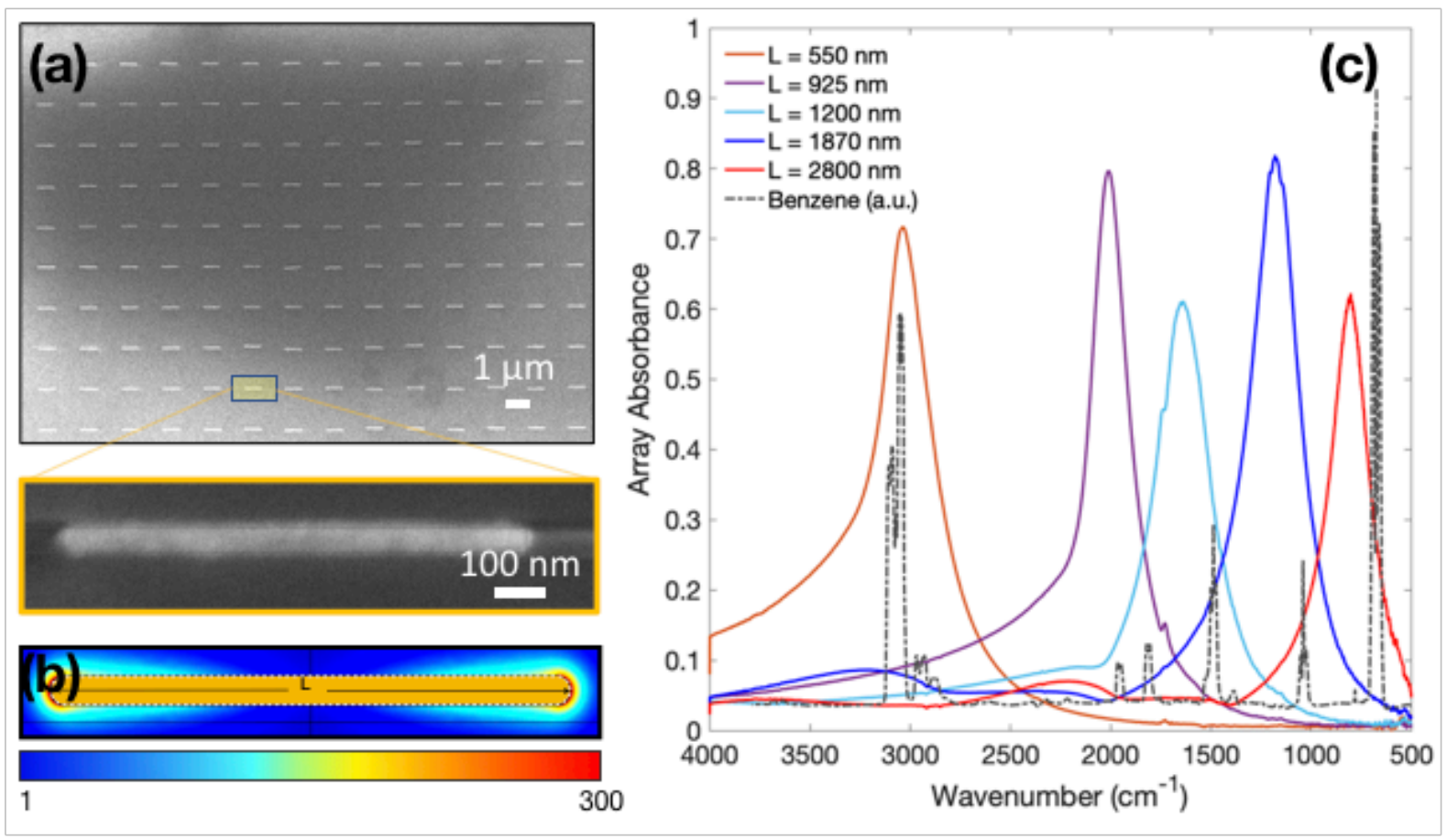

Figure 3: (a) SEM image of a resonant nano-antenna array composed of phased gold nanorods. (b) Field distribution at resonance, normalized by the incident field. (c) IR spectra of nanoantenna array of different length overlapped with benzene spectrum (dotted spectrum).

fabricated arrays revealing nanorod size and pattern periodicity, which is very important to have an optimal response in phase scattering. Figure $3 \mathrm{~b}$ shows the electromagnetic field distribution simulated at resonance conditions, with a maximum near field enhancement in the order of 300 . The experimental IR response of a set of arrays for a beam polarized along the long axis of the nanorods, is presented in Figure 3c, showing the tuneability of resonant features across a wide range of wavelengths, by mainly adjusting the nanorod length (see SI for further description). If the beam was polarized perpendicularly to the antenna axis (short length), no resonant features were visible, in accordance with theoretical predictions (Figure S3); a clean ZnSe substrate was used as the reference.

\subsection{Detection of benzene traces}

Next, a FAU zeolite coating was used to trap and concentrate the targeted analyte in close proximity of the nano-antennas where the field enhancement is the highest (see Figure $3 b$ ). 


\section{WILEY-VCH}

The coating with an average thickness of $200 \mathrm{~nm}$ was prepared on the nano-antenna array via drop-casting. Figure 4a displays an SEM image of the sample overlaid with elemental compositions maps obtained via energy dispersive X-ray spectroscopy (EDX). A crack in the zeolite coating allows us to see the underlying gold nano-antenna array. Individual elemental composition maps are presented in Figure $4 \mathrm{~b}$. As expected $\mathrm{Si}, \mathrm{O}$ and $\mathrm{Al}$ coexist within the nanocrystals.

We selected a nano-antenna array with nanorod length of $1200 \mathrm{~nm}$, to assess the enhancement of the benzene band around $1480 \mathrm{~cm}^{-1}$. We chose to focus on this band because the spectral
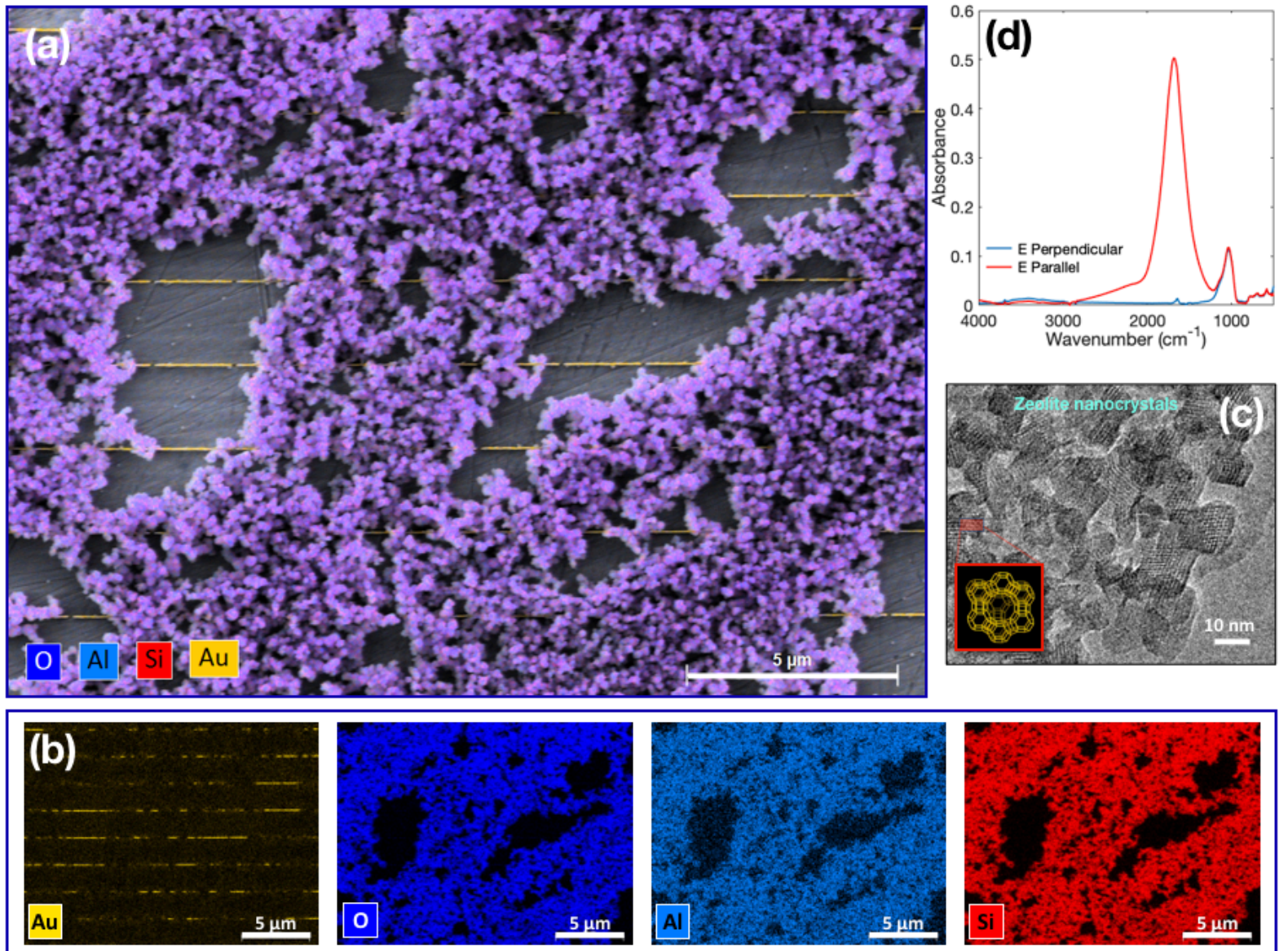

Figure 4: (a) SEM image of a resonant nano-antenna array coated with FAU zeolite nanocrystals. The color corresponds to elemental distribution obtained by overlaying the EDX maps shown in (b). The corresponding EDX spectrum is presented in Figure S5. (c) TEM image of FAU zeolite nanocrystals. The inset shows the FAU zeolite structure represented by the supercharge constructed by 6 sodalite cages connected via double-six rings (d) IR spectrum of $1200 \mathrm{~nm}$ long nano-antenna array coated with zeolite nanocrystals for parallel (red) and perpendicular polarization (blue), showing the resonance and the zeolite fingerprint. 


\section{WILEY-VCH}

feature of benzene was not influenced by other vibrational changes due to the coatingbenzene interaction.

After deposition, a vibrational mode $\left(1045 \mathrm{~cm}^{-1}\right)$ characteristic of FAU zeolite appears in the spectrum presented in Figure 4d (blu solid line). This mode lays outside the nano-antennas resonance and as expected it is not amplified.

The nano-antenna-zeolite was mounted in a gas cell in the optical path of IR light to assess its performance; mass flow controllers were used together with calibrated permeation tubes to obtain low concentrations of gases. The sample response upon exposure to $25 \mathrm{ppb}$ of benzene is shown in Figure 5a and for different exposure times (IR light is polarised parallel to the nanorods long axis) in Figure 5b. A strong effect on the IR spectrum is observed within a few minutes on the bands that correspond to the interaction of benzene with the zeolite (940-1100 $\left.\mathrm{cm}^{-1}\right)$. The spectral variation in the region of $1000 \mathrm{~cm}^{-1}$ and $1700 \mathrm{~cm}^{-1}$ is consistent with what observed for a FAU coating without antennas after benzene exposure (Figure 2) as described above. It's interesting to note that the band around $1700 \mathrm{~cm}^{-1}$ corresponding to water is modulated by the nano-antennas resonance, as evidenced by the response for perpendicular polarisation presented in Figure S8.

Within 10 minutes, the characteristic aromatic $\mathrm{C}=\mathrm{C}$ stretching peak of benzene $\left(1480 \mathrm{~cm}^{-1}\right)$ is already above the noise $(>3 \sigma)$. The change of the intensity of the band at $1480 \mathrm{~cm}^{-1}$ as a function of time is presented in Figure 5c, both for parallel and perpendicular polarization.

In the framework of temporal coupled mode theory, the introduction of a loss channel as a result of the coupling with molecular vibrations (dark mode- weak compared to the nanoantennas mode) causes a perturbation of the coupling with external radiation. In undercoupled systems (radiation losses smaller than intrinsic absorption losses) it reduces absorption (electromagnetically induced transparency), while in over-coupled systems it 


\section{WILEY-VCH}

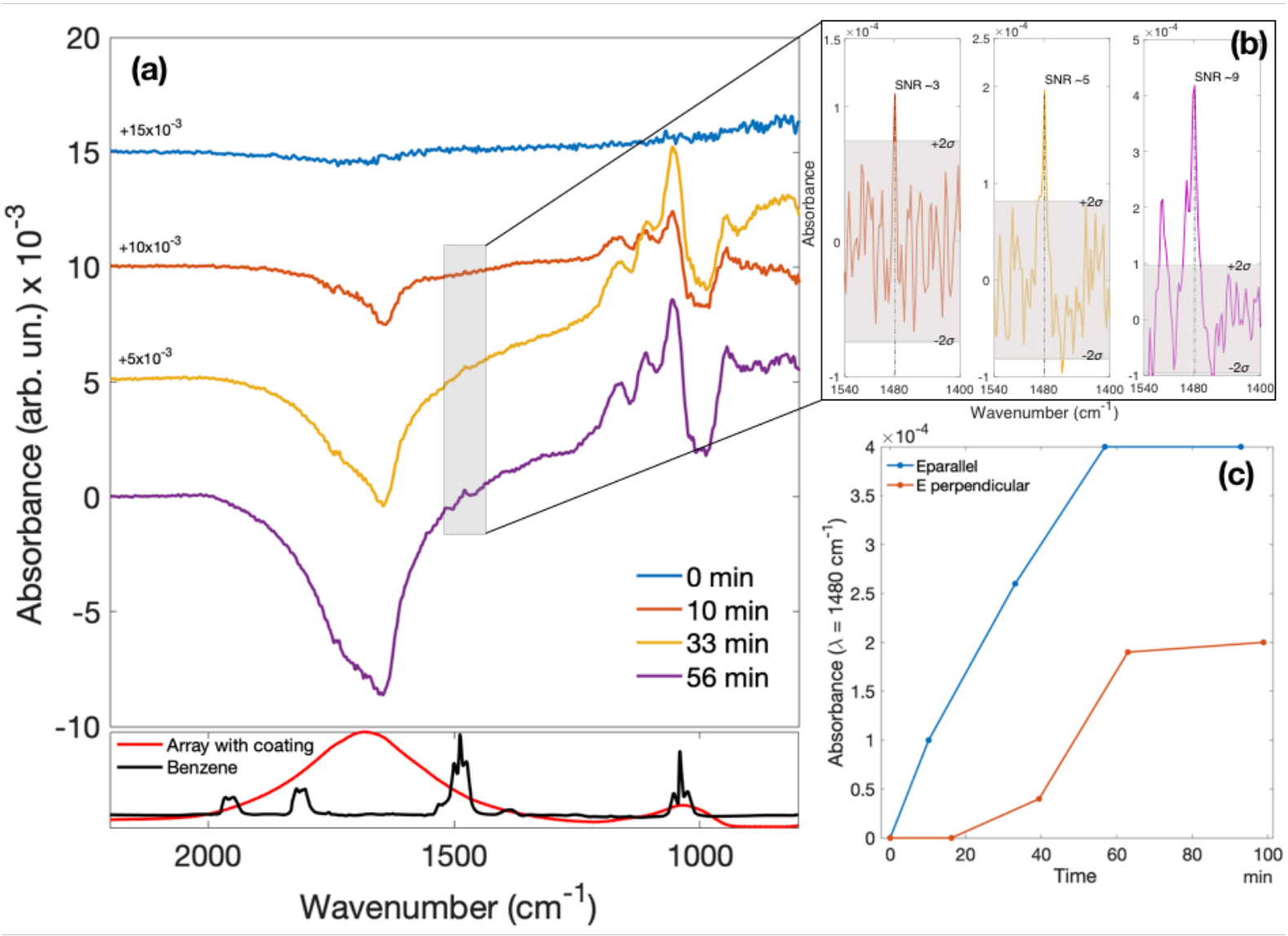

Figure 5: $(\mathrm{a}, \mathrm{b})$ IR response of nano-antenna-zeolite device upon exposure to $25 \mathrm{ppb}$ of benzene at different time intervals. The spectrum at time 0 (after $\mathrm{N}_{2}$ flushing, before benzene exposure) is used as a reference; the sample was placed in the cell during the entire experiment, to avoid artefacts due to inhomogeneity in the sample (nano-antenna and/or coating) and spatial dependence of the IR beam. (c) Intensity of the peak at $1480 \mathrm{~cm}^{-1}$ as a function of time for parallel (blue) and perpendicular (red) polarisation. Nano-antenna length: $1200 \mathrm{~nm}$.

increases it (electromagnetically induced absorption), as the ratio between radiative to nonradiative losses is reduced, driving the system towards the condition of critical coupling, that maximises absorption [10]. Considering that in our system radiation losses are larger than intrinsic absorption losses (over-coupled, see Figure S9), the increase in absorbance upon benzene exposures (compared to perpendicular polarization) is consistent with predictions obtained in the framework of temporal coupled mode theory.

The boost in sensitivity given by the resonant interaction of nano-antennas with the vibrational modes of benzene trapped within the near field enhancement volume is demonstrated in Figure 5. The concerted action of the FAU zeolite coating and the resonant nano-antennas enabled us to attain performance equivalent to a $\sim 120 \mathrm{~m}$ long optical path (see 


\section{WILEY-VCH}

SI for further details). As shown in Figure 4d, the optical response of nano-antennas is strongly anisotropic. This enabled us to evaluate the individual contribution of the nanozeolite coating and SEIRA on the very same sample, by looking at the ratio of absorbance between parallel and perpendicular polarization in Figure 5c. The nano-antennas amplified the absorbance by a factor $\sim 3$ as a consequence of resonant coupling, despite the overlap between the array resonance and the benzene mode was not optimal.

Although, the contribution of nano-antennas is modest when compared to the zeolite concentration factor, it enabled us to detect $25 \mathrm{ppb}$ of benzene in $<10$ minutes as opposite to $\sim 1$ h. In addition to faster detection time, a larger SNR is obtained, which is especially important in the fabrication of portable devices.

Considering that the coating thickness was larger than the near-field enhancement, which is located near the nanorod ends, most of the coating did not interact with nano-antennas. As a consequence, the results shown in Figure 5c suggest that even thinner coatings could be employed to boost response time without sacrificing sensitivity. This, combined with nanophotonic designs may improve the use of the substrate real estate in terms of field enhancement, and could further improve the performance.

\section{Conclusions}

We showed that by combining the nanozeolite concentrating coating with specifically designed IR nano-antennas was possible to use SEIRA for enhancing the light-matter interaction with the gas molecules used in this study (benzene and toluene). As a consequence, the detection of benzene down to $25 \mathrm{ppb}$ was possible. In addition to already mentioned strategies, the detection limit and SNR can be easily improved by using a $\mathrm{N}_{2}$ 


\section{WILEY-VCH}

cooled MCT detector as opposed to the DGTS detector used in this work, or by optimizing the thickness of the zeolite coating. Optimization of the zeolite coating with the emphasis on the thickness, chemical composition and porosity will be considered in the near future. Further perspectives for future works include the use of core-shell architectures [26] with the nano-antennas being the resonant core uniformly coated by a zeolite shell, and ultimately, employing monocrystalline nano-antennas to reduce ohmic losses applying the procedure previously reported [27]. This findings would have important effect in the design of portable systems for efficient detection of low concentrated gas pollutants in the atmosphere, especially in urban areas. Furthermore, the possibility to detect VOC in low concentrations can enforce guidelines regarding air quality standards.

\section{Supporting Information}

Supporting Information is available from the Wiley Online Library or from the author

\section{References}

Received: ((will be filled in by the editorial staff))

Revised: ((will be filled in by the editorial staff)) Published online: ((will be filled in by the editorial staff))

[1] L. Spinelle, M. Gerboles, G. Kok, S. Persijn, T. Sauerwald, Sensors-Basel 2017, 17.

[2] D. Dregely, F. Neubrech, H. G. Duan, R. Vogelgesang, H. Giessen, Nat Commun 2013, 4.

[3] T. Etzkorn, B. Klotz, S. Sorensen, I. V. Patroescu, I. Barnes, K. H. Becker, U. Platt, Atmos Environ 1999, 33, 525-540.

[4] R. Lu, G. P. Sheng, W. W. Li, H. Q. Yu, Y. Raichlin, A. Katzir, B. Mizaikoff, Angew Chem Int Edit 2013, 52, 2265-2268.

[5] M. Sieger, B. Mizaikoff, Analytical Chemistry 2016, 88, 5562-5573.

[6] C. R. Young, N. Menegazzo, A. E. Riley, C. H. Brons, F. P. DiSanzo, J. L. Givens, J. L. Martin, M. M. Disko, B. Mizaikoff, Anal Chem 2011, 83, 6141-6147.

[7] F. Neubrech, C. Huck, K. Weber, A. Pucci, H. Giessen, Chem Rev 2017, 117, 5110-5145.

[8] X. Yang, Z. Sun, T. Low, H. Hu, X. Guo, F. J. Garcia de Abajo, P. Avouris, Q. Dai, Adv Mater 2018, 30, e1704896.

[9] F. Neubrech, A. Pucci, T. W. Cornelius, S. Karim, A. Garcia-Etxarri, J. Aizpurua, Phys Rev Lett 2008, 101.

[10] R. Adato, A. Artar, S. Erramilli, H. Altug, Nano Lett 2013, 13, 2584-2591. 


\section{WILEY-VCH}

[11] T. Neuman, C. Huck, J. Vogt, F. Neubrech, R. Hillenbrand, J. Aizpurua, A. Pucci, J Phys Chem C 2015, 119, 26652-26662.

[12] R. Adato, H. Altug, Nat Commun 2013, 4.

[13] W. Chen, F. Cazier, F. Tittel, D. Boucher, Appl Opt 2000, 39, 6238-6242.

[14] R. Sur, Y. Ding, R. B. Jackson, R. K. V. Hanson, Applied Physics B 2019, 125.

[15] Y. H. Chang, D. Hasan, B. W. Dong, J. X. Wei, Y. M. Ma, G. Y. Zhou, K. W. Ang, C. Lee, Acs Appl Mater Inter 2018, 10, 38272-38279.

[16] X. Y. Chong, Y. J. Zhang, E. W. Li, K. J. Kim, P. R. Ohodnicki, C. H. Chang, A. X. Wang, Acs Sensors 2018, 3, 230-238.

[17] H. Zhou, X. D. Hui, D. X. Li, D. L. Hu, X. Chen, X. M. He, L. X. Gao, H. Huang, C. Lee, X. J. Mu, Adv Sci 2020.

[18] A. Jentys, R. R. Mukti, H. Tanaka, J. A. Lercher, Micropor Mesopor Mat 2006, 90, 284-292.

[19] S. Mintova, J. P. Gilson, V. Valtchev, Nanoscale 2013, 5, 6693-6703.

[20] T. Babeva, H. Awala, M. Vasileva, J. El Fallah, K. Lazarova, S. Thomas, S. Mintova, Dalton T 2014, 43, 8868-8876.

[21] M. J. Hwang, W. G. Shim, D. W. Ryu, H. Moon, J Chem Eng Data 2012, 57, 701-707.

[22] H. Awala, J. P. Gilson, R. Retoux, P. Boullay, J. M. Goupil, V. Valtchev, S. Mintova, Nat Mater 2015, 14, 447-451.

[23] N. Y. Chen, J Phys Chem-Us 1976, 80, 60-64.

[24] L. Novotny, Phys Rev Lett 2007, 98.

[25] D. Weber, P. Albella, P. Alonso-Gonzalez, F. Neubrech, H. Gui, T. Nagao, R. Hillenbrand, J. Aizpurua, A. Pucci, Opt Express 2011, 19, 15047-15061.

[26] B. Sciacca, S. A. Mann, F. D. Tichelaar, H. W. Zandbergen, M. A. van Huis, E. C. Garnett, Nano Lett 2014, 14, 5891-5898.

[27] B. Sciacca, A. Berkhout, B. J. M. Brenny, S. Z. Oener, M. A. van Huis, A. Polman, E. C. Garnett, Adv Mater 2017, 29. 


\section{WILEY-VCH}

The table of contents entry should be 50-60 words long and should be written in the present tense. The text should be different from the abstract text.

Javier Nunez ${ }^{1}$, Arjen Boersma1 , Julien Grand ${ }^{2}$, Svetlana Mintova ${ }^{2}$ and Beniamino Sciacca ${ }^{3}$

Thin functional zeolite layer supported on infrared resonant nano-antennas for the detection of benzene traces

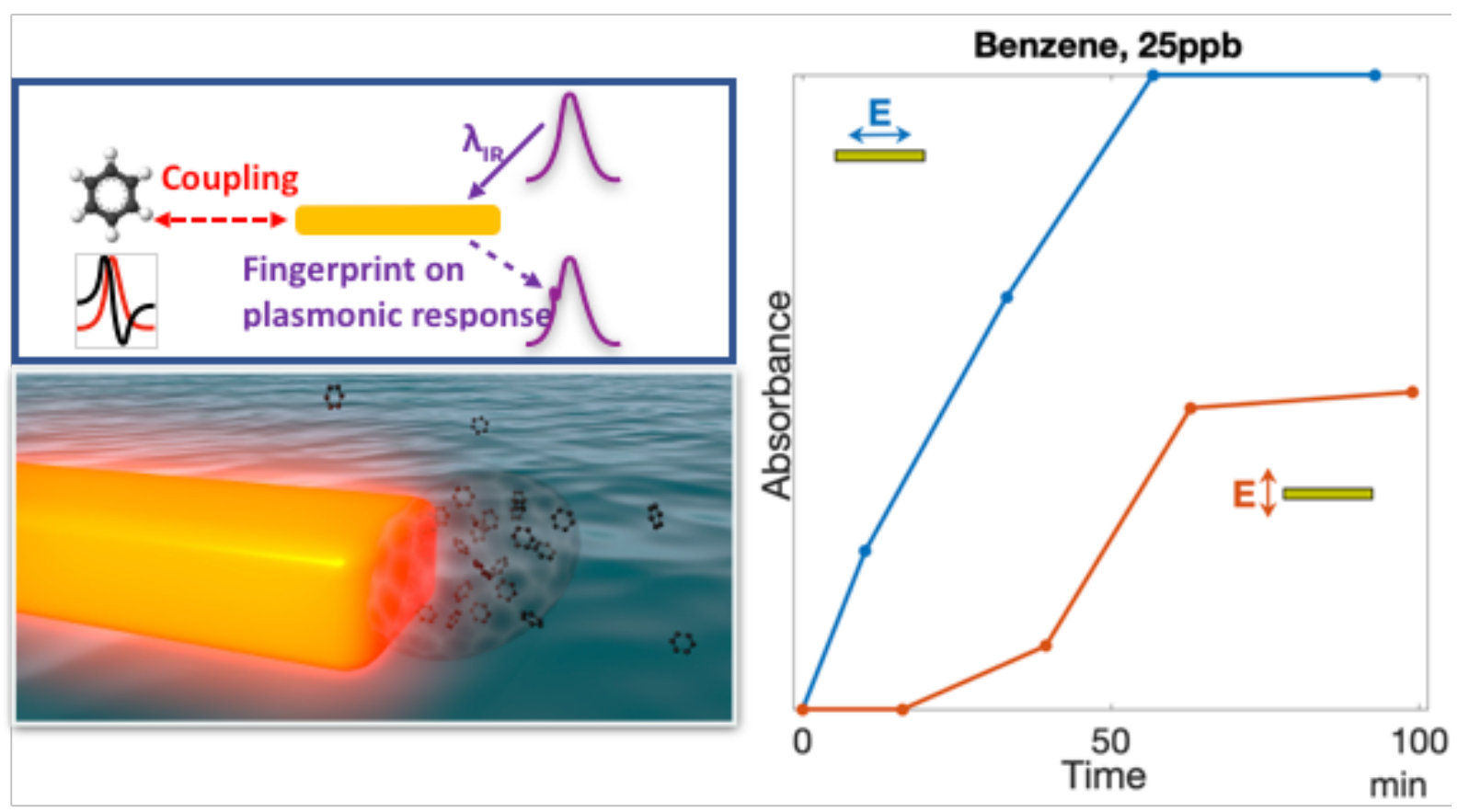

The concerted action of infrared resonant nano-antennas with an ultra-thin zeolite concentrating coating, enables unprecedented rapid detection of benzene traces ( $25 \mathrm{ppb})$. In this hybrid material, the zeolite coating effectively traps VOCs analytes nearby the hotspots of nano-antennas, thus enabling Surface Enhanced Infrared Absorption (SEIRA). Sensitivity equivalent to a $\sim 120 \mathrm{~m}$ long optical path is achieved in a $\sim 200 \mathrm{~nm}$ layer. 


\section{WILEY-VCH}

\section{Supporting Information}

\section{Thin functional zeolite layer supported on infrared resonant nano-antennas for the detection of benzene traces}

Javier Nunez ${ }^{1}$, Arjen Boersma1, Julien Grand ${ }^{2}$, Svetlana Mintova ${ }^{2}$ and Beniamino Sciacca ${ }^{3 *}$

\section{Experimental Setup}

The experimental setup consists of a Fourier-Transform Infrared (FTIR) spectrophotometer Thermofisher Nicolet 6700, with a DGTS detector, equipped with an Auxiliary Experimental Module (AEM) in which a custom gas cell system is installed. The gas cell is a modified commercially available Pike Technologies Short Path Gas Cell, that on one end has a $\mathrm{KBr}$ window and, on the other, a custom metal lid with two round orifices where samples are placed, as shown in Figure S1.
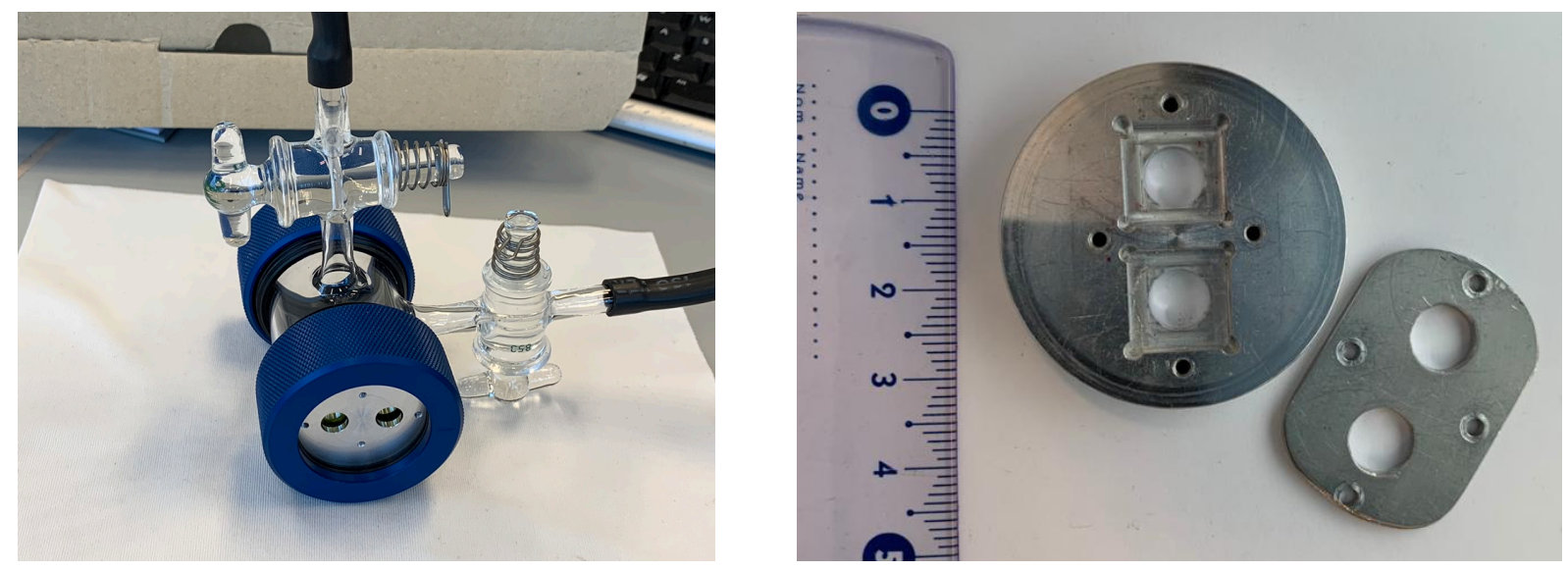

Figure S1. Gas cell featuring a custom metal lid with two orifices where the samples are placed and fixed.

Both orifices are designed so that the samples (square shaped and bigger than the orifice) can fit in square dips on the inner surface of the lid, mounted on viton O-rings. For their fixation, a small metal cover, also provided with two identical round orifices, is placed in turn on new O-rings on top of the samples, and screwed onto the main lid. This way, the samples form a "sandwich" between the gas cell lid and the internal metal cover, where they are "suspended" with O-rings on both faces. Thus, a good gas sealing of the system is guaranteed.

A calibrated permeation tube is placed in the permeation device's oven, and this is set at $60{ }^{\circ} \mathrm{C}$ with a constant flow of nitrogen. The calibrated permeation tube has a mass loss of $345 \mathrm{ng} / \mathrm{min}$ at $60{ }^{\circ} \mathrm{C}$. By tuning the nitrogen flow with mass flow controllers a desired concentration of benzene in the nitrogen atmosphere is obtained. As an example, a nitrogen flow of $4 \mathrm{~L} / \mathrm{min}$ results in a benzene concentration of $\sim 86 \mathrm{ng} / \mathrm{L}$. Taking into account the molar mass of benzene $(78.11 \mathrm{~g} / \mathrm{mol})$ and the molar volume of nitrogen at $60{ }^{\circ} \mathrm{C}(27.34 \mathrm{~L} / \mathrm{mol})$, the benzene fraction can be calculated as $86 \times 27.34 / 78.11 \sim 30 \times 10^{-9}=30 \mathrm{ppb}$.

To assess the accuracy, the absorbance was measured for a range of benzene concentrations (in a $14.66 \mathrm{~m}$ optical path cell) and was compared with the absorbance calculated from the absorption coefficient [1]. The results, shown in Figure S2, indicate that the target concentration is accurate and that the benzene generation system has a linear behaviour over a wide range of concentration. 

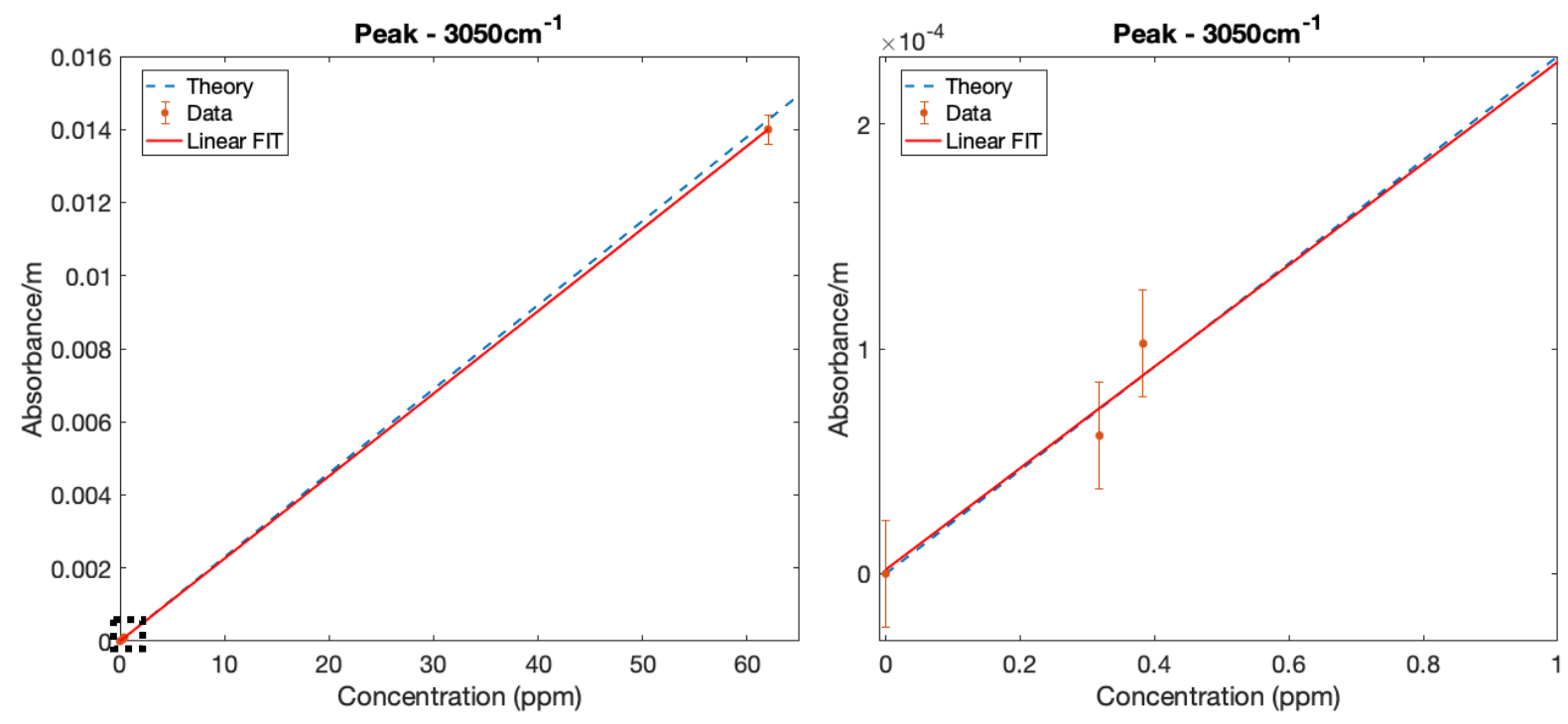

Figure S2. Characterisation of the concentration accuracy. Left: the absorbance (normalised by the optical path) is plotted as a function of the benzene concentration. The dots represent experimental data, the solid line the linear fit for all the data points over the whole range, and the dashed line corresponds to the theoretical absorbance $/ \mathrm{m}$ calculated from the absorption coefficient $\left(2 \times 10^{-4} / \mathrm{ppm} / \mathrm{m}\right)$ for the peak at $3050 \mathrm{~cm}^{-1}$. Right: zoomed view of the normalised absorbance at low concentration of benzene.

\section{Spectral acquisition}

A background was always taken once the spectrum was stable in dry conditions prior to benzene exposure, for the two polarizations. The spot diameter was 7 millimeters, and the spot position was identical for the background and posterior measurements to enable a consistent analysis. The number of scans was set at 64 , the data spacing was set to $1.928 \mathrm{~cm}^{-1}$ and the optical velocity was set to the lowest possible value $(0.1581)$.

A polariser in the optical path allows us to tune the polarization of the IR beam.

\section{Nano-antenna design.}

Several nanoantenna designs were developed to match the vibrational fingerprint of benzene in the range 3-15 $\mu \mathrm{m}$. Due to the complexity of the system, numerical simulations were performed to find, for a given resonance wavelength, an optimal configuration (highest field enhancement) in terms of nanorod size and array lattice parameters. The commercial package COMSOL multiphysics was used to run the optimisations, which aimed at maximising the local field enhancement of resonances that overlapped with benzene vibrational fingerprints. The arrays were modelled on a $\mathrm{ZnSe}$ substrate, and only a quarter of the system was simulated as a result of symmetry consideration. A plane wave was launched in the system and the reflected, transmitted and absorbed spectrum was calculated, along with the near field distribution.

A total of five arrays was designed, to match five major modes of benzene (see table below). The height and width of nanorods were inversely proportional to the field enhancement, in accordance with calculations reported in literature [2]. However, to allow for reproducible fabrication of such arrays, the width was fixed at 65 $\mathrm{nm}$ and the height at $35 \mathrm{~nm}$. ZnSe was selected as the substrate to allow for transmission measurements in the range of interest (3-15um), and because, compared to other substrates as undoped $\mathrm{Si}$ or $\mathrm{Ge}$, it has a smaller refractive index $(\sim 2.3)$ which reduces the amount of field localised inside the substrate itself.

The periodicity of the array in the direction along the long axis of the rod (X axis) was fixed to have an end-toend distance between adjacent rods of $\sim 100 \mathrm{~nm}$, which allows for near field interaction between the rods (the smaller the gap the higher the field enhancement) and for the deposition of a concentrating coating in the gap. 


\section{WILEY-VCH}

The periodicity of the array in the Y-direction and the length of the nanorods were the variables used to optimise the field enhancement for the given resonance wavelengths. The periodicity in the Y-direction allowed us to have constructive interference of the scattered field, as mentioned above, to maximize the enhancement. In order to suppress grating modes, and have only forward scattered light, the periodicity in the Y-direction needs to be below the first grating order, that is approximately $\sim \lambda_{\text {res }} / \mathrm{n}_{\text {substrate. }}$ Optimal conditions (perfectly in phase scattered fields) normally occur just below the first grating order. However, from the macroscopic point of view, a further decrease in periodicity from one hand negatively affects the maximum local field enhancement (scattered fields are less and less in phase), but from the other hand allows having more nanorods and therefore more hotspots (spots with enhanced electromagnetic radiation). Since the accurate design and optimisation are quite cumbersome, as one needs to take into account the size of respective mode volumes and the interaction with coating and molecules, a different approach was chosen. Typically, the arrays resonant at small energies (8.5 um and $12.5 \mathrm{um}$ ) were designed to have a larger number of nanorods instead of a perfectly phased condition. This is because the experimental deviation from ideal conditions, such as for example substrate flatness/roughness, was estimated to occur more likely at a very large Y periodicity, therefore decreasing the experimental field enhancement. Instead, for the arrays resonant at higher energy ( $3.3 \mathrm{um}, 5$ and $6.2 \mathrm{um}$ ), the phased condition design was followed.

The design parameters for the arrays corresponding to the spectra shown in Figure 2 are summarized in Table 1. The maximum field enhancement (magnitude) was in the order of 200-300, depending on the array configuration.

\begin{tabular}{|c|c|c|c|c|}
\hline & L $(\mathbf{n m})$ & Dx $(\mathbf{n m})$ & Dy $(\mathbf{n m})$ & Resonance $(\mathbf{c m}-\mathbf{1})$ \\
\hline Chip 1 & 550 & 700 & 1300 & 3030 \\
\hline Chip 2 & 925 & 1075 & 1900 & 2000 \\
\hline Chip 3 & 1200 & 1350 & 2000 & 1610 \\
\hline Chip 4 & 1870 & 2020 & 2000 & 1180 \\
\hline Chip 5 & 2800 & 2960 & 2900 & 940 \\
\hline
\end{tabular}

Table 1. Physical parameters of nano-antennas fabricated on the ZnSe substrate. $L$ is the nanorods length (oriented along the X-axis), Dx and Dy represent the lattice parameters of the array. The width (W) is $60 \mathrm{~nm}$.

\section{Nano-antenna fabrication.}

Fabrication was performed via e-beam lithography by the company Nanophab based at TU/e (Technical University of Eindhoven). Briefly, a ZnSe substrate $\left(30 \times 30 \mathrm{~mm}^{2}\right)$ was first coated with a conductive polymer and subsequently with an electron sensitive resist. After calibrating the correct amount of electrons to dose for exposing a certain feature, the structures were written on a large area $\left(6.5 \times 6.5 \mathrm{~mm}^{2}\right.$ for each array). After exposure and development, $35 \mathrm{~nm}$ of Au were deposited on the sample, followed by a lift-off procedure. Then, a protective layer of PMMA $(600 \mathrm{~nm})$ was deposited prior to dice the substrate into $10 \times 10 \mathrm{~mm}^{2}$ pieces. After dicing, PMMA was removed with acetone and IPA. 


\section{WILEY-VCH}

\section{Nano-antenna array characterization.}

Figure S2 shows the experimental absorbance band of Chip 2 (resonance at $2000 \mathrm{~cm}^{-1}$ ) for parallel and perpendicular polarisation.

The small peak present at $1730 \mathrm{~cm}^{-1}$ is due to the $\mathrm{C}=\mathrm{O}$ stretching mode of some residual PMMA on the substrate. This peak can be used to estimate the enhancing capabilities of the nanoantennas. An average amplification of $\sim 40$ is obtained by calculating the ratio between the parallel and orthogonal polarisation for the peak at $1730 \mathrm{~cm}^{-1}$. The filling fraction of the array is $\mathrm{L}_{\mathrm{x}} \mathrm{W} / \mathrm{D}_{\mathrm{x}} \mathrm{D}_{\mathrm{y}}=2.7 \%$ (percentage of surface occupied by nanorods). However, the nanoantennas enhance only molecular vibrations of molecules that lie in the near vicinity, due to the nature of the near field distribution that decays exponentially [3]. One can therefore assume that only molecules that lie within $\sim 100 \mathrm{~nm}$ at most of the nanoantennas extremities are excited with an amplified field; taking into account the array geometry, a local effective enhancement $>4000$ can be estimated $\left(40 * \mathrm{Dx} * \mathrm{Dy} / 2 \cdot 100^{2}\right)$.

As one can see from the field plot in Figure 1, this estimation is fairly conservative, since the field decays quite rapidly from the nanoantennas surface, and therefore the actual local enhancement is underestimated. A more precise estimation can be performed by calculating the overlap integral of the field distribution with the molecule absorption, which is beyond the scope of this paper.

It is interesting to note that the shape of the band at $1730 \mathrm{~cm}^{-1}$ is different among the arrays shown in Figure 1. In fact, in some arrays it appears as a peak (increase absorbance), while in some other arrays it appears as a dip (decreased absorbance), or it has an asymmetric (Fano-like) shape. This phenomenon was shown to be dependent on the relative spectral position of the molecular vibrational mode with the nanoantenna resonance. It is related to the phase between the molecular oscillator and the nanoantenna resonator [4]. An oxygen plasma cleaning step was employed to remove such PMMA traces before to deposit the zeolite coating.

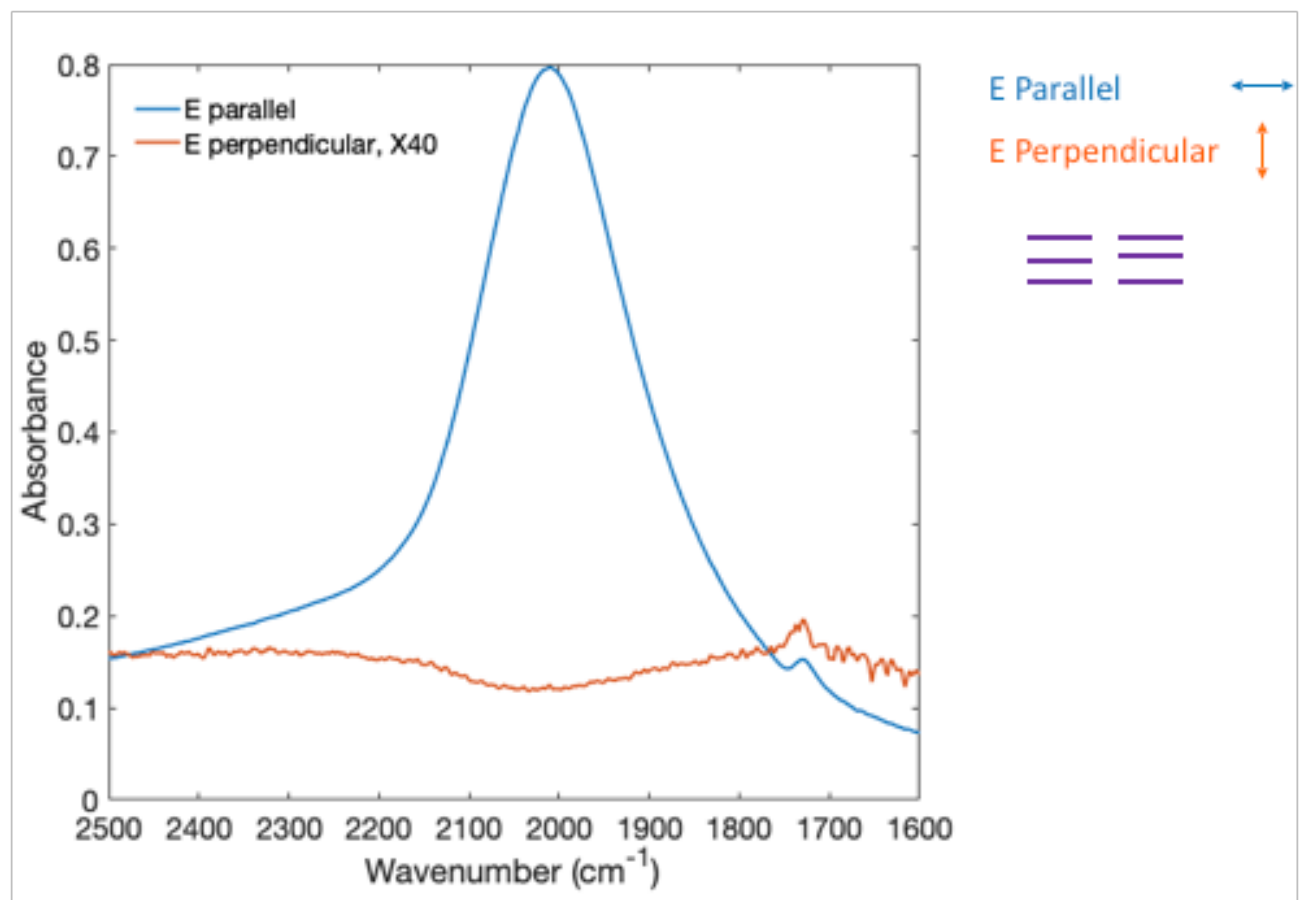

Figure S3: Comparison between parallel and perpendicular polarisation of the incoming IR light. The small peak observed at $1730 \mathrm{~cm}^{-1}$ is due to residual PMMA, and is used to assess the performance of the array. Note that the curve for perpendicular polarisation is magnified by a factor 40 to allow the comparison. Also the acquisition time (optical velocity and number of scans) needed to resolve the peak for perpendicular polarisation was substantially higher than that for the parallel polarisation due to the smaller signal to noise ratio. 


\section{WILEY-VCH}

\section{Selection of nanosized zeolite}

Within the vast database of zeolites materials, different structures (EMT, EMT, FAU, MFI) were considered to test the response towards species such as benzene. The choice was based on the following arguments:

1) Hydrophobicity. Due to the non-polar nature of target molecules such as benzene, zeolites materials with a more hydrophobic character were expected to be able to concentrate such molecules to a larger extent. Furthermore, for a sensor to work in uncontrolled environments, it is important to reduce as much as possible the interference of other agents such as humidity. The degree of hydrophobicity is controlled by the $\mathrm{Si} / \mathrm{Al}$ ratio, with higher ratios results in more hydrophobic materials.

2) Pore diameter. The pore diameter of the zeolite material is a crucial factor determining the effectiveness of the coating to adsorb the species of interest. Considering that the size of the benzene molecule is in the order of $\sim 0.5 \mathrm{~nm}$, structures with pores larger than $0.5 \mathrm{~nm}$ were selected.

3) Availability as nano-sized crystals. Due to the system design it is important to coat the nanoantennas with a zeolite film in the order of $100 \mathrm{~nm}$, to exploit at best the field enhancement effect. Therefore bottom-up synthesis of nano-sized zeolites crystals was selected. This allows: i) low temperature deposition (no damage on the nanoantennas) and ii) conformal coating with a tuneable thickness.

4) Affinity. Another important aspect that was taken into consideration was the affinity of the zeolite material towards benzene. This relates to the chemical interactions that takes place inside the pores when a benzene
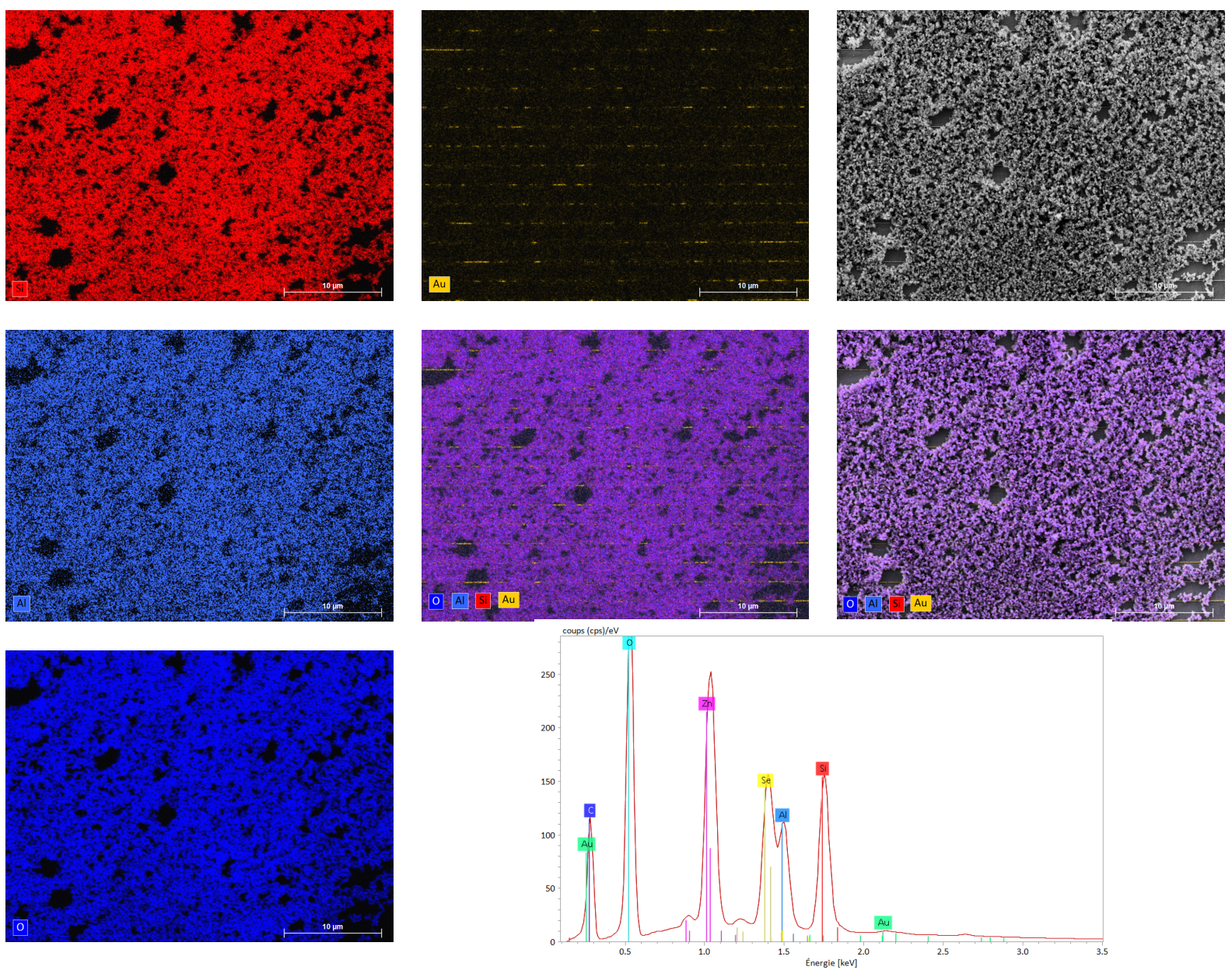

Figure S4: Elemental compositions mapping for a FAU coating on nanoantennas and EDX analysis 


\section{WILEY-VCH}

molecule enters. In other words it is important that at very low partial pressure the coating is capable of adsorbing the target species, and therefore that possesses a large Henry's constant. Furthermore, depending on the metal loading the zeolites, a better concentration factor can be observed at low partial pressure. Ag and $\mathrm{Cu}$ loading have been shown to coordinate extremely well with benzene.

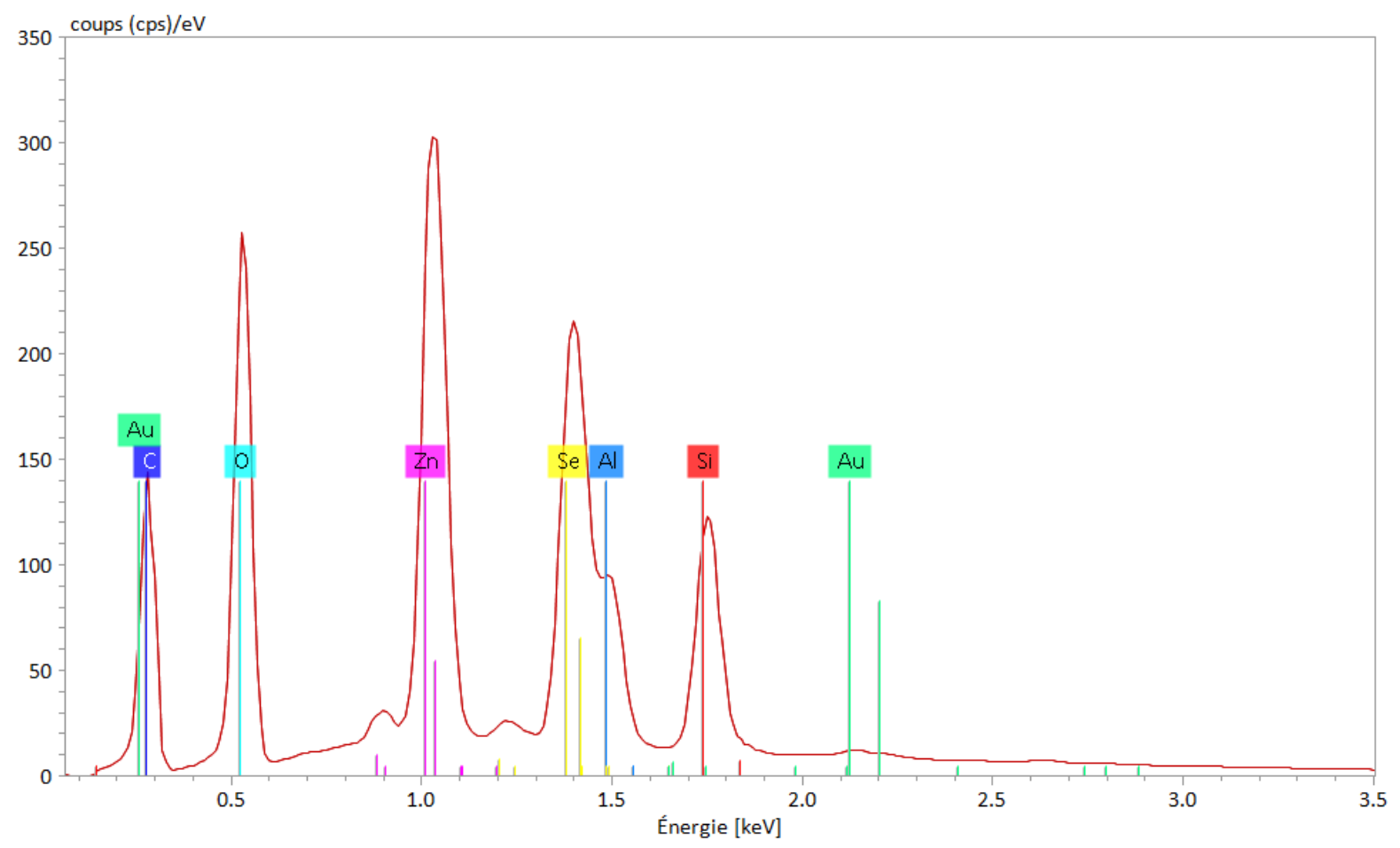

Figure S5: EDX spectrum corresponding to elemental maps shown in Figure 4.

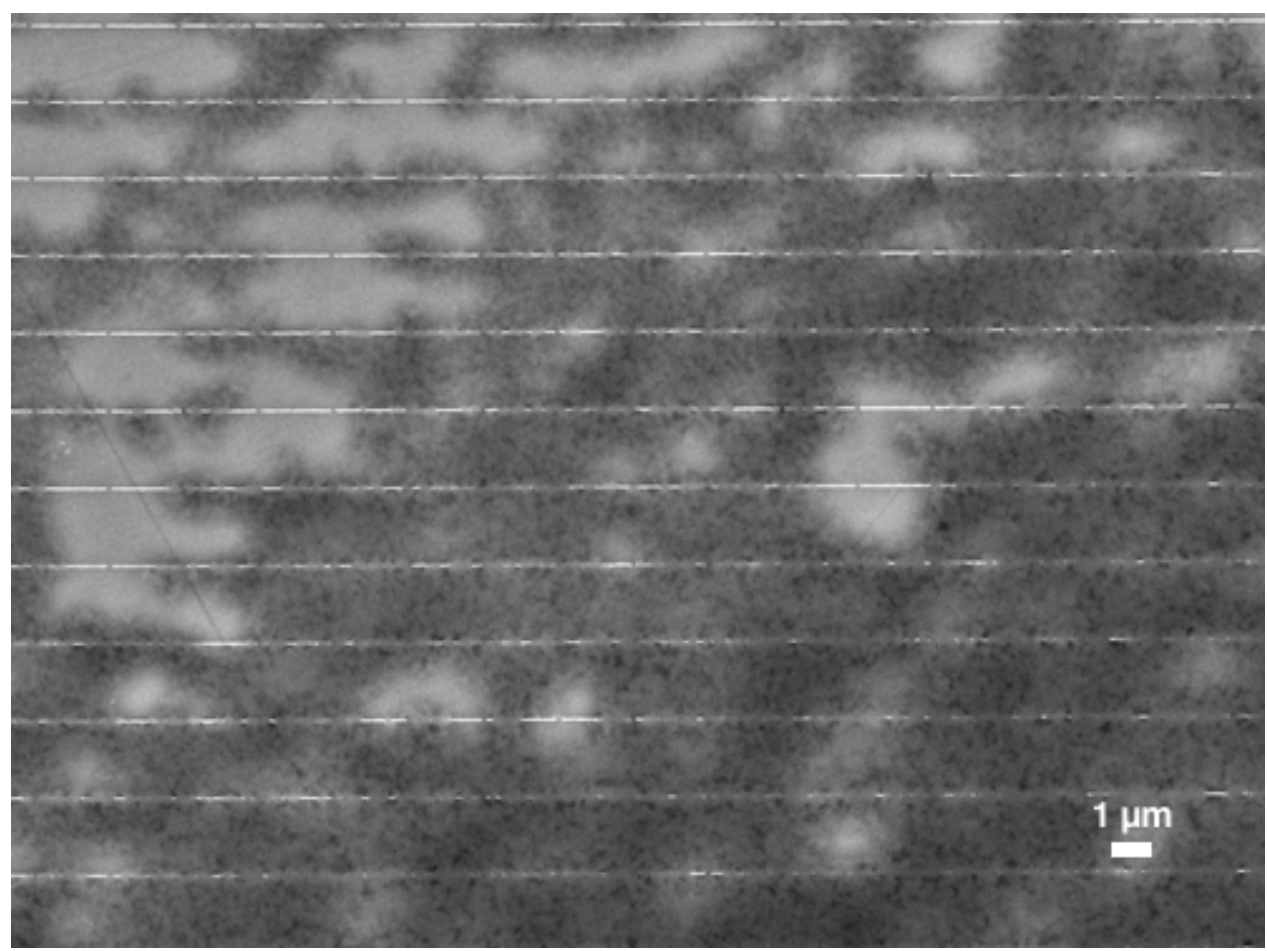

Figure S6: Backscattered electrons image of a nanoantenna array coated with FAU zeolite crystals. 


\section{WILEY-VCH}

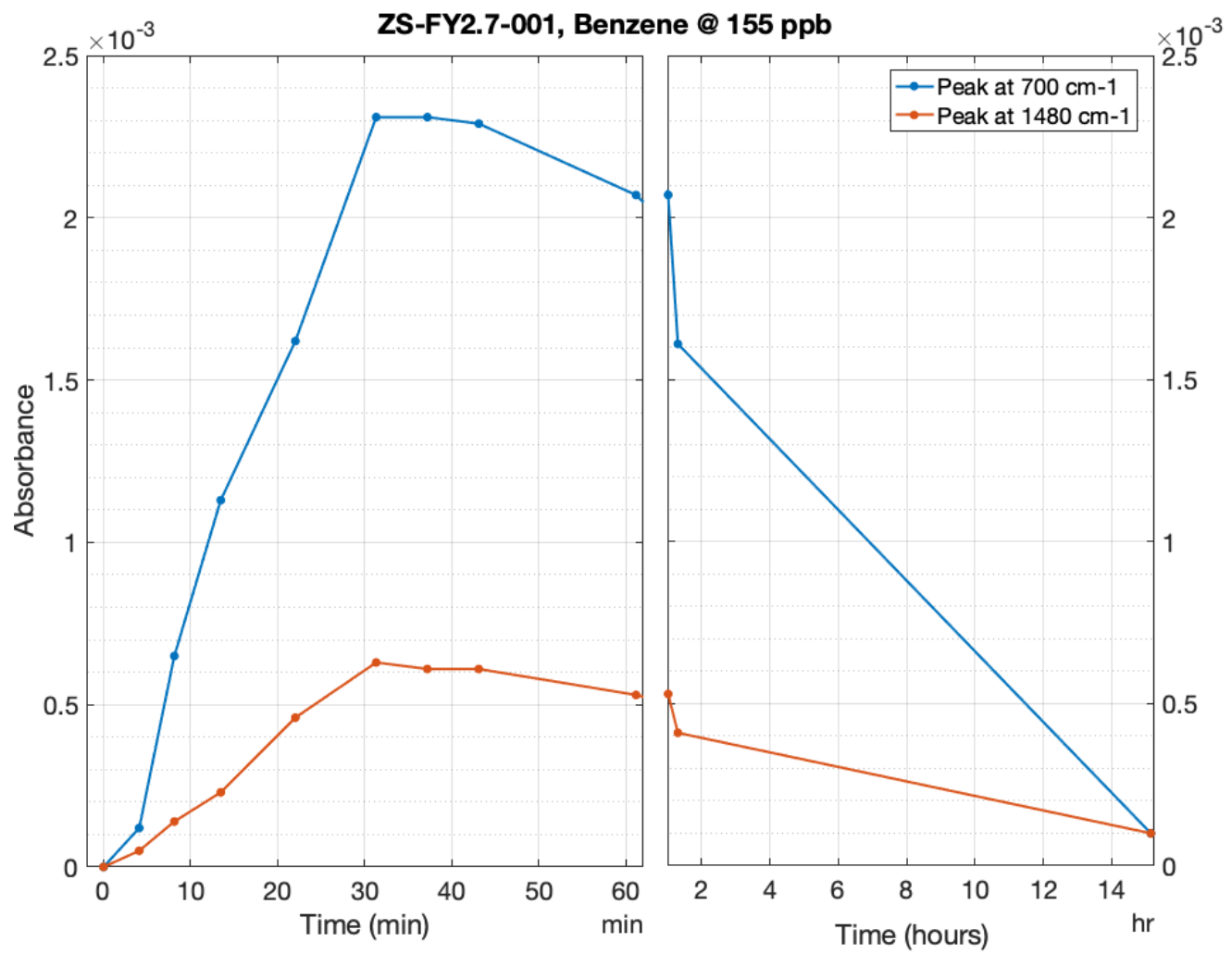

Figure S7: Kinetic response to a $155 \mathrm{ppb}$ benzene (1-30 min) and flushing in dry nitrogen (30 $\mathrm{min}-15 \mathrm{~h})$ to show the reversibility at room temperature.

\begin{tabular}{|c|c|c|c|c|c|c|}
\hline $\begin{array}{c}\text { Structur } \\
\mathrm{e}\end{array}$ & $\begin{array}{c}\text { Pores } \\
(\AA)\end{array}$ & Framework & $\begin{array}{l}\text { Si/Al } \\
\text { ratio }\end{array}$ & $\begin{array}{c}\text { Availability } \\
\text { as } \\
\text { nanosized }\end{array}$ & Hydrophobicity & Affinity \\
\hline EMT & $6-7$ & & 1.2 & Yes & Low & $\begin{array}{l}\text { High, } \\
\text { can be } \\
\text { doped } \\
\text { by Ag }\end{array}$ \\
\hline \multirow{2}{*}{ FAU } & \multirow{2}{*}{7} & & 1.6 & Yes & Low & High \\
\hline & & & 2.7 & Yes & Medium & High \\
\hline
\end{tabular}




\section{WILEY-VCH}

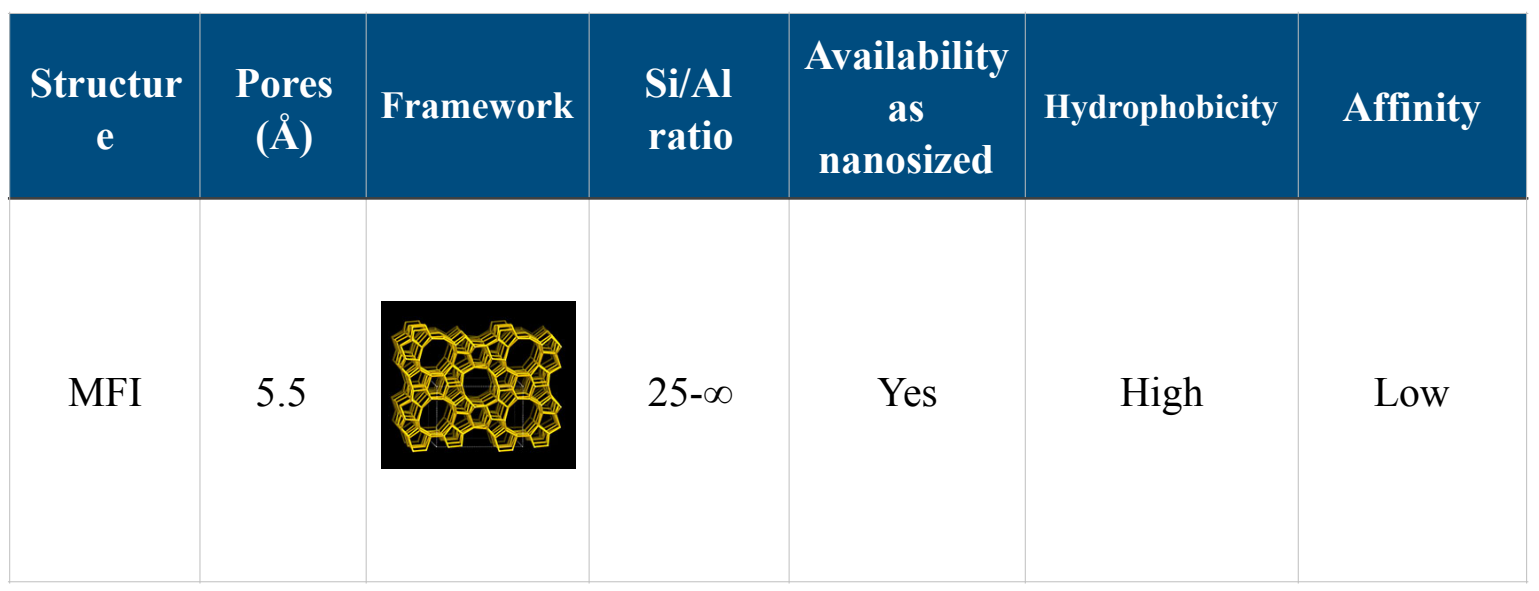

Table 2: Properties of zeolite coatings.

\section{Nanozeolite synthesis and deposition.}

Nanozeolites were synthesized with a procedure described previously [5]. The FAU zeolite crystals were further stabilized in colloidal coating suspensions. Next, the suspensions were sonicated for 5 minutes, in order to make them homogeneous, and subsequently an aliquot was taken and drop-casted onto the substrate and let it dry.

\section{Characterisation of the zeolite coating}

To characterize the performance of different coating when exposed to benzene and toluene, the samples were mounted on the gas cell and the absorbance was monitored as a function of concentration and/or time. Prior to exposure, the glass cell was purged with dry nitrogen until a stable spectrum was obtained over a window of $\sim 1$ h. The transmission through the coating in nitrogen environment was used as background to reference the spectra. The reference measurement was taken exactly in the same location of the exposure measurements, as the flow cell is not displaced for the entire duration of the experiment. This avoids the appearance of artefacts related to inhomogeneity in the zeolite coating

The absorbance measured depends on the effective amount of Benzene in the optical path of the IR beam. In a scenario without coating, for a given benzene concentration, the measured Absorbance follows Lambert-Beer's law, and depends on the gas cell optical path $(5 \mathrm{~cm}$ in our setup) and on the molar extinction coefficient of benzene for that specific molecular transition (peak energy), which is an intrinsic feature of the target molecule. When a coating is present, the target molecules accumulate inside the coating itself, therefore yielding a higher absorbance than that without a coating. The amount of benzene/toluene that can be adsorbed inside the zeolite coating depends, as mentioned in previous sections, on the chemical interactions at a molecular level, that define the equilibrium constant of the system. To isolate the contribution of coating affinity and assess performance, the thickness of the deposited coating was measured with a profilometer and averaged along different line scans. This allowed us to normalise the absorbance by the thickness, and calculate a concentration factor (defined in Equation 1), that was used to compare different coatings with different thicknesses. The meaning of this factor relates to the amount of target molecules per unit length, and therefore to the ability of the coating to concentrate a certain species.

Without coating the absorbance of benzene in the gas phase with a concentration of $62 \mathrm{ppm}$, in the $5 \mathrm{~cm}$ gas cell was measured to be $0.3 \cdot 10^{-3}$ for the peak at $1485 \mathrm{~cm}^{-1}$, that is consistent with the absorption coefficient $\left(1 \times 10^{-4 /}\right.$ 


\section{WILEY-VCH}

$\mathrm{ppm} / \mathrm{m}$ ). For comparison, when the coating was present the absorbance at $1480 \mathrm{~cm}^{-1}$ was $0.2 \cdot 10^{-3}$ at $25 \mathrm{ppb}$ (Figure $5 \mathrm{c}$ ), therefore enabling a sensitivity $\sim$ three orders of magnitude higher than the prediction from LambertBeer's law.

The absorbance was monitored as a function of time for a fixed concentration, to study the kinetics of the system. This allowed to assess the loading of the coating and to estimate the reversibility (release of molecules from the coating when no species are present in the outside environment).

Figure S7 shows the variation of absorbance for two characteristic benzene peaks after exposure to benzene $(\mathrm{t}=1-30 \mathrm{~min})$ and flushing in dry nitrogen $(30 \mathrm{~min}-15 \mathrm{~h})$, showing the coating reversibility at room temperature

\section{Performance summary}

Equation 1 was used to estimate the concentration factor using the peak at $1480 \mathrm{~cm}^{-1}$. As an example, for a FAUY2.7 with $\mathrm{t}_{\text {coat }} 200 \mathrm{~nm}$ an absorbance of $\mathrm{A}_{\text {sat }}=0.2 \cdot 10^{-3}$ was measured for a benzene concentration of $\mathrm{C}_{0}=25 \mathrm{ppb}$, while without coating an absorbance of $0.3 \cdot 10^{-3}$ was measured for a benzene concentration of $\mathrm{C}_{\mathrm{ref}}=62 \mathrm{ppm}$ for a $\mathrm{d}_{\text {cell }}=5 \mathrm{~cm}$ optical path of the gas cell. Therefore $\boldsymbol{\alpha}=6 \cdot 10^{8}$. The concentration factor calculated for the mode at $1812 \mathrm{~cm}^{-1}$ and that at $691 \mathrm{~cm}^{-1}$ was $\sim 4.7 \cdot 10^{8}$ and $\sim 7 \cdot 10^{8}$ respectively, showing that it does not vary significantly.

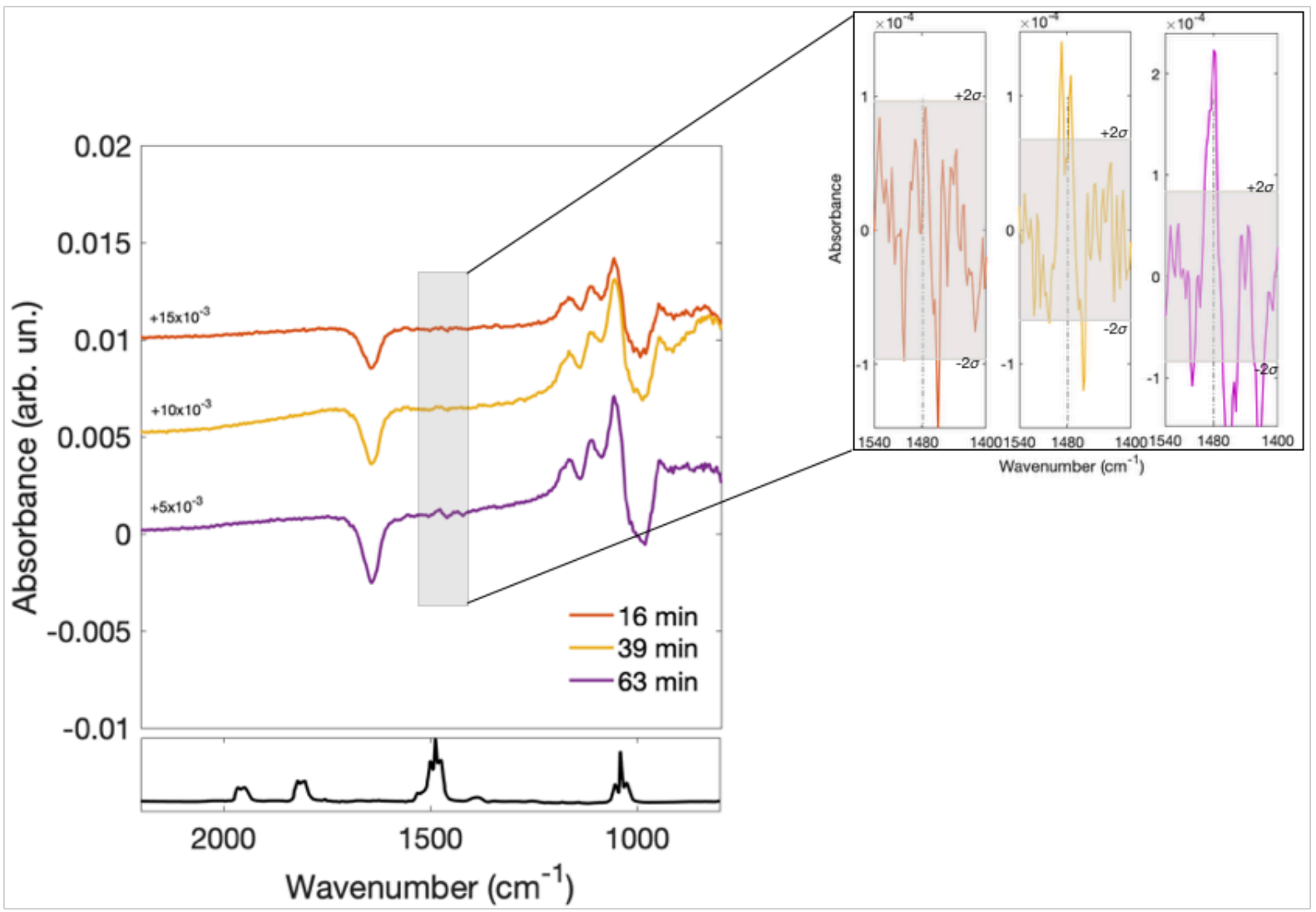

Figure S8: IR spectra corresponding to Figure 5c for perpendicular polarisation (exposure to 25ppb of benzene) 


\section{WILEY-VCH}

\section{FDTD simulations}

FDTD simulations were performed with the commercial software Lumerical. The interaction of a polarised plane wave with the nano-antenna array was simulated with periodic boundary conditions on $\mathrm{x}, \mathrm{y}$ and perfectly absorbing layers on z. The array geometry is defined in Table 1 (Chip 6). A refractive index of 2.4 was used for the ZnSe substrate. The calculated optical properties are shown in Figure S9 (a).

The array is overcoupled as evidence by Figure S9 (b). The fit corresponds to the following parameters: $\gamma_{a e}=113.3 \mathrm{~cm}^{-1} ; \gamma_{a 0}=76.49 \mathrm{~cm}^{-1} ; \omega_{\text {res }}=1645 \mathrm{~cm}^{-1} ; R_{\text {substrate }}=0.169$ (for $\mathrm{ZnSe}$ ).

Where $\gamma_{a e}=113.3 \mathrm{~cm}^{-1}$ represents losses related to coupling to external radiation, while $\gamma_{a 0}=76.49 \mathrm{~cm}^{-1}$ intrinsic material absorption. Since $\gamma_{a e}>\gamma_{a 0}$ the system is overcoupled $[4,6]$.
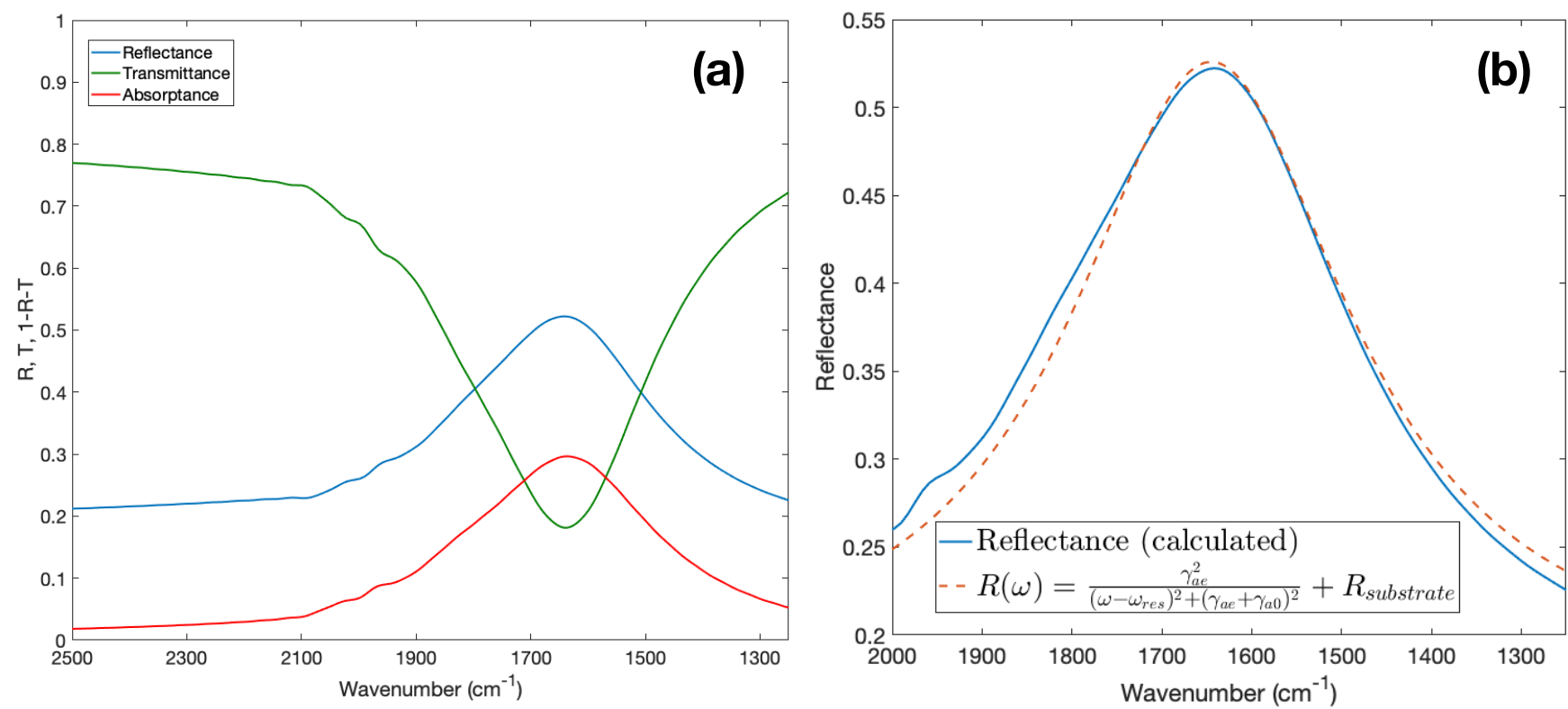

Figure S9: FDTD simulations of the nano-antenna array response (left), FIT of the Reflectance according to temporal coupled mode theory 


\section{References}

[1] P. Chu et al., The NIST Quantitative Infrared Database, J. Res. Natl. Inst. Stand. Technol. 1999, 104, 59.

[2] J. Aizpurua, et al., Optical properties of coupled metallic nanorods for field-enhanced spectroscopy, Phys Rev B 2005, 71,235420.

[3] D. Dregely, et al., Vibrational near-field mapping of planar and buried three-dimensional plasmonic nanostructures, Nat Commun 2013, 4, 2237.

[4] R. Adato, et al., Engineered Absorption Enhancement and Induced Transparency in Coupled Molecular and Plasmonic Resonator Systems, Nano Lett 2013, 13, 2584.

[5] H. Awala, et al., Template-free nanosized faujasite-type zeolites, Nat Mater 2015, 14

[6] R. Adato, H. Altug, In-situ ultra-sensitive infrared absorption spectroscopy of biomolecule interactions in real time with plasmonic nanoantennas Nat Commun 2013, 4, 2154. 